Research Article

\title{
Earthquake Protection of Existing Structures with Limited Seismic Joint: Base Isolation with Supplemental Damping versus Rotational Inertia
}

\author{
Dario De Domenico (iD) and Giuseppe Ricciardi \\ Department of Engineering, University of Messina, 98166 Sant'Agata, Messina, Italy \\ Correspondence should be addressed to Dario De Domenico; dario.dedomenico@unime.it
}

Received 28 August 2017; Revised 11 December 2017; Accepted 12 December 2017; Published 19 March 2018

Academic Editor: John Mander

Copyright (C) 2018 Dario De Domenico and Giuseppe Ricciardi. This is an open access article distributed under the Creative Commons Attribution License, which permits unrestricted use, distribution, and reproduction in any medium, provided the original work is properly cited.

\begin{abstract}
Existing civil engineering structures having strategic importance, such as hospitals, fire stations, and power plants, often do not comply with seismic standards in force today, as they were designed and built based on past structural guidelines. On the other hand, due to their special importance, structural integrity of such buildings is of vital importance during and after earthquakes, which puts demands on strategies for their seismic protection. In this regard, seismic base isolation has been widely employed; however, the existing limited seismic joint between adjacent buildings may hamper this application because of the large displacements concentrated at the isolation floor. In this paper, we compare two possible remedies: the former is to provide supplemental damping in conventional base isolation systems and the latter consists in a combination of base isolation with supplemental rotational inertia. For the second strategy, a mechanical device, called inerter, is arranged in series with spring and dashpot elements to form the so-called tuned-mass-damper-inerter (TMDI) directly connected to an isolation floor. Several advantages of this second system as compared to the first one are outlined, especially with regard to the limitation of floor accelerations and interstory drifts, which may be an issue for nonstructural elements and equipment, in addition to disturbing occupants. Once the optimal design of the TMDI is established, possible implementation of this system into existing structures is discussed.
\end{abstract}

\section{Introduction}

Passive vibration control systems of civil engineering structures and infrastructures are of utmost importance in earthquake-prone regions to mitigate or reduce damage potential due to the shaking ground. Even more importantly, seismic protection is imperative for those structures whose integrity during and after earthquakes is of vital importance for civil and social purposes, including hospitals, fire stations, schools, barracks, power plants, and so forth. Nevertheless, in many cases such structures present structural deficiency and do not comply with the requirements of seismic standards in force today, as they were designed and built according to the past structural guidelines that were not as severe as the current regulations. Consequently, seismic protection and retrofitting strategies of these structures are highly desirable, which should do not interfere with the operational and functionality aspects of the building.

In this regard, seismic base isolation $[1,2]$ has been widely employed along with energy dissipation systems $[3,4]$. The lengthening of the first-mode period combined with the damping features provided by the base isolation system (BIS) significantly reduces the earthquake-induced forces in the superstructure, which can be designed to remain in the elastic regime. As a counter effect, most of the earthquake-induced displacement demand is concentrated at the isolation floor, which undergoes large displacements (whose magnitude depends on the design value of the BIS effective period). This has important implications in the retrofitting costs, as large-size isolators should be adopted that can accommodate the required displacements, in addition to considering costly flexible connections for utilities 
(e.g., waterworks, gas fittings, and electrical conduits). In most cases, this implies a complete refurbishment of the existing, more rigid pipelines, which significantly increases the overall retrofitting costs. In addition, an adequate separation distance between adjacent buildings, also called seismic joint, must be present in order to prevent mutual collisions or structural pounding [5]. The latter aspect may limit the base isolation applications in those cases involving a limited space between adjacent buildings, as the actual seismic joint may easily be exceeded by the design displacement of the BIS. Therefore, the goal is to find a seismic protection strategy such that the superstructure will experience little damage, but at the same time, the overall displacement of the structure remains limited to within a reasonably acceptable threshold, thus not exceeding the seismic joint. Another problem that is often encountered in seismic base isolation is the vulnerability to long-period ground motions that might severely increase the lateral displacements of the isolators and cause long-lasting vibrations due to the resonance effects [6], in a similar fashion to high-rise flexible-frame buildings $[7,8]$.

These shortcomings have motivated the development of improved versions of the conventional base isolation scheme. The most straightforward remedy for reducing the BIS displacement is to increase the isolation damping or to provide supplemental damping. Although this strategy does reduce the excessively large displacements in the BIS, it may increase the interstory drifts and floor accelerations in the superstructure [9]. In addition to disturbing occupants, this may be an issue for nonstructural elements and equipment that could be of valuable importance in the above mentioned strategic buildings (e.g., control centers, real-time acquisition data centers, and so on). As an alternative, a hybrid control strategy combining the conventional base isolation system with supplemental rotational inertia is proposed in this paper. In particular, the BIS is coupled with the so-called tuned-mass-damper-inerter (TMDI). The latter system, by analogy with the tuned mass damper (TMD) [10], consists of mass, spring, and damper elements that are installed in series with a novel type of device, the inerter [11]. Details of this system will be discussed below.

This paper compares the above two alternative strategies for the seismic retrofitting of existing buildings, namely, seismic base isolation with supplemental damping versus seismic base isolation with supplemental rotational inertia. Optimal tuning parameters of the TMDI are detected within a probabilistic framework, by considering the stochastic nature of earthquake ground motions and solving a nonlinear optimization problem. The earthquake ground motion is modelled as a Gaussian process in the frequency domain through the use of the power spectral density (PSD) function. The influence of soil characteristics is investigated by introducing three PSD functions representatives of firm, medium, and soft soil conditions. Based on the optimal parameters found, a possible implementation scheme with regard to existing structures is proposed. Some numerical applications demonstrate advantages and disadvantages of the two vibration control strategies with regard to the structural control of existing structures, not only in terms of displacement but also in terms of transmitted forces and other response indicators such as the base shear, the interstory drifts, and the floor accelerations, in order to evaluate the overall performance of these seismic protection systems.

\section{Inerter and Tuned-Mass-Damper-Inerter (TMDI)}

The inerter element was introduced by Smith [11] in 2002 to complete the force-current analogy between mechanical and electrical networks. In particular, the inerter has been proposed as the mechanical counterpart of the capacitor concept in electrical systems. If $u_{1}$ and $u_{2}$ are the displacement coordinates of the two terminals connected by the inerter, the following relationship holds for the ideal linear inerter:

$$
F=b\left(\ddot{u}_{1}-\ddot{u}_{2}\right),
$$

where the constant of proportionality $b$ is called inertance and has dimensions of mass; thereby, it has been sometimes termed "apparent mass." From (1), it is shown that the internal force of the inerter is proportional to the relative acceleration of its two terminals, similarly to the damper and spring elements whose internal force is proportional to the relative velocity and displacement, respectively.

As schematically shown in Figure 1, the inerter can be realized through a two-terminal device that consists of a combined arrangement of a rack, pinions, gears, and a flywheel. In this figure, $m_{f}$ is the mass of the flywheel, $r_{i}$ and $r_{\mathrm{pi}}$ denote the radius of gears and pinions, respectively, $r_{f}$ is the radius of the flywheel, and $r_{\mathrm{pf}}$ indicates the radius of the flywheel pinion. Linear movement of the rack generates rotational movement in gears and flywheel, and the inertance of this system can be expressed as

$$
b=m_{f} \frac{r_{f}^{2}}{r_{\mathrm{pf}}^{2}}\left(\prod_{i=1}^{n} \frac{r_{i}^{2}}{r_{\mathrm{pi}}^{2}}\right),
$$

where $n$ is the number of gears. Therefore, this system is based on the concept of rotational inertia. Alternative realizations involve a rotary ball screw and a flywheel to convert axial movement into rotary displacements. The inerter has also been employed in the automotive sector under the name of $J$-damper for Formula 1 racing car suspensions [12]. By adjusting the gearing ratios or simply introducing additional gears, it is possible to attain very high values of the inertance $b$, without implying a large amount of physical mass $m_{f}$. In principle, any desired value of $b$ can be obtained with a device of reasonably compact form. Such mass amplification effect is the key feature of this system and makes it appealing for vibration control purposes: it has been demonstrated that the apparent mass can be designed to be orders of magnitude (up to 200 times) higher than the physical mass of the device. This peculiarity has been exploited in the context of enhancing the performance of tuned mass dampers (TMDs). In particular, as sketched in Figure 2 the TMDI consists of an enhanced version of TMD (of mass $m_{t}$, spring stiffness $k_{t}$, and viscous damping $c_{t}$ ) 


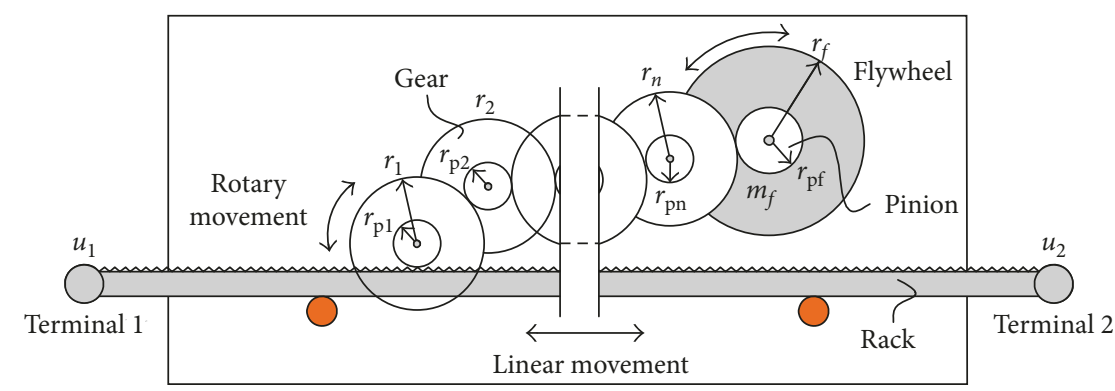

FIGURE 1: Schematic model of the inerter with rack and pinion mechanism.

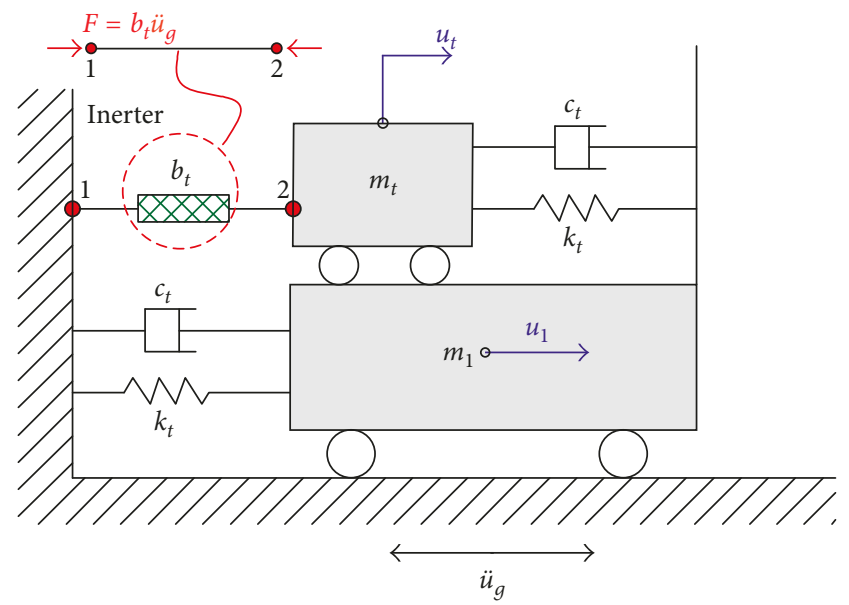

FIGURE 2: Sketch of the tuned-mass-damper-inerter (TMDI).

in which an inerter device, schematically represented by its inertance, is placed in between the TMD mass and the ground (points 1 and 2). For the above mentioned characteristics, the TMDI can be viewed as a lower mass and more effective alternative to the traditional TMD, where the device inertance plays the role of the TMD mass [13-16]. While very large mass amounts are required for the TMD to achieve an acceptable vibration reduction, effective vibration control can be attained by the TMDI with reasonably limited physical mass. Moreover, while the TMD stroke is usually quite large, the vibration reduction is attained by the TMDI without implying excessive stroke of the additional mass.

Besides the optimal design of the TMDI, which is described below in terms of its dynamic characteristics (stiffness, damping, etc.), there are a few practical considerations related to the functionality of the inerter that deserve commentary. Unlike mechanical networks involving frequent activity of the rack and pinion mechanism, in earthquake engineering, it is expected that the inerter actually works just for few seconds, that is, during the seismic event. The horizontal accelerations transmitted by the shaking ground during this short time generate resistive forces in the inerter by accelerating the internal wheels. However, there may be an issue of backfeeding energy into the structure when the building begins to slow down, unless an internal mechanism decouples the rotational mass to prevent this phenomenon. This energy is certainly transferred from the inerter to the TMD; therefore, an adequate space in the building is necessary to allow this stroke. However, this energy is not returned to the superstructure, since the inerter is not directly connected to it, but just to the TMD mass. There is an additional filter, consisting of the TMD spring and damper elements, which significantly reduce this backfeeding energy to the superstructure at the end of the earthquake. This reduction can be seen by the observation of the time history of the response at the end of the seismic event (see below). Further investigation is certainly needed to carefully analyze these phenomena in more detail, which is beyond the main scope of this paper.

\section{Base Isolation with Supplemental Rotational Inertia via TMDI}

In the context of hybrid control strategies, applications of BIS in conjunction with a TMD attached immediately above or below the isolation floor of the building have been extensively discussed in the literature [17-20]. It has been found that by attaching a TMD to the BIS, a certain reduction of the displacement demand of a base-isolated structure is achieved, depending on the TMD mass. Conversely, applications of inerter-based devices in the context of base-isolated structures are really few [21-25]. To the authors' best knowledge, the dynamic layout of the BIS coupled with the TMDI has never been studied in the literature as a seismic retrofitting strategy of existing structures, which constitutes the main novelty of the present research work. In particular, a practical implementation of this strategy is here proposed via the use of two sets of isolators. Besides the conventional isolators, an auxiliary set of medium-to-high damping isolators are located underneath the structure; the latter playing the role of damper and spring elements of the TMD. Another important aspect that has never been presented in previous studies is the optimal design of this combined BIS + TMDI system and, above all, the influence of the soil conditions on the tuning parameters, which is here dealt with within a wide parametric study. Due to the stochastic nature of earthquake ground motions, such optimal parameters of the TMDI are sought within a probabilistic framework by minimization of 


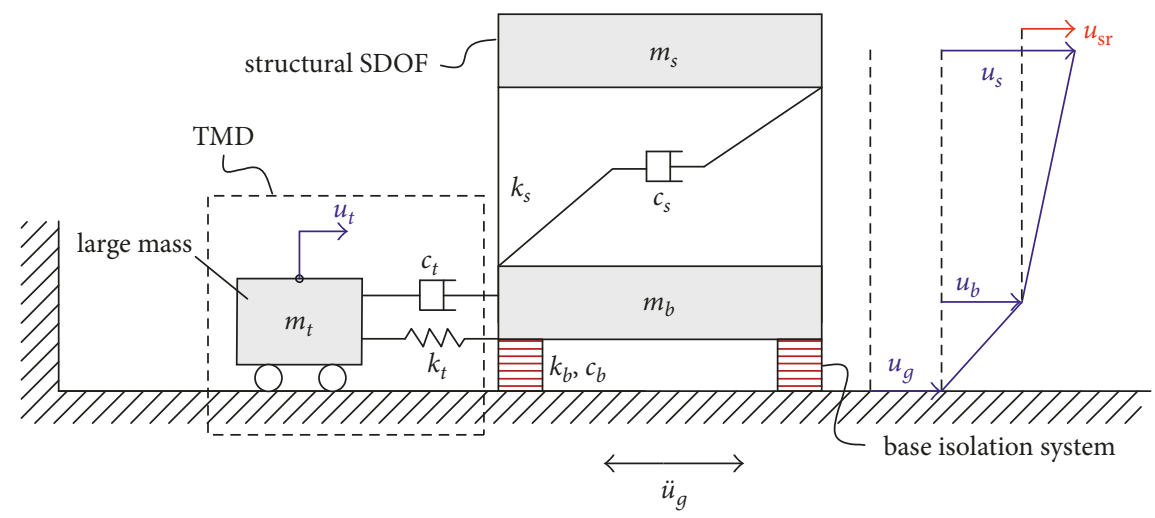

(a)

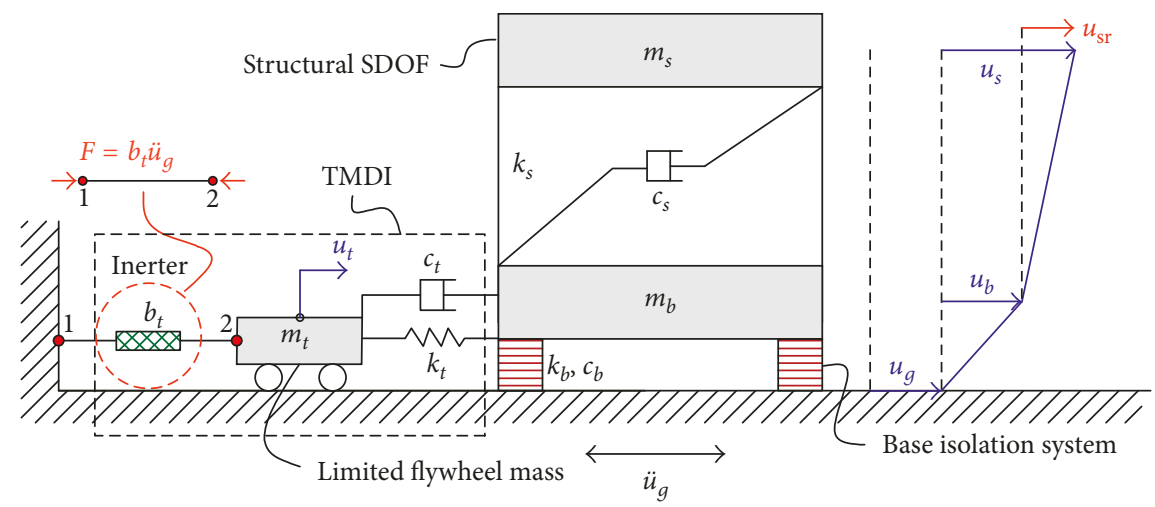

(b)

FIGURE 3: Sketch of base-isolated structural SDOF with attached TMD (a) and with attached TMDI (b).

the displacement variance. Furthermore, the performance of this system is assessed in terms of a variety of response indicators that are useful and of practical importance for design engineers, such as the base shear, interstory drifts, and floor accelerations.

Due to the proportionality of the generated force of the inerter to the relative acceleration of its two terminals as per (1), this element can easily be incorporated into the equations of motion as the inertance will only appear in the mass matrix. In particular, reference is made to the simple sketch shown in Figure 3, wherein a single degree of freedom (SDOF) structural system is base isolated and equipped with a TMDI attached to the isolation level. The three degrees of freedom (DOFs) are the BIS displacement $u_{b}$ relative to the ground, the superstructure displacement $u_{\text {sr }}$ relative to the $\mathrm{BIS}$, and the TMDI displacement $u_{t}$ relative to the ground. Linear dynamical systems are considered with an equivalent linear viscous damping idealization for BIS, TMDI, and superstructure. The equations of motion of this simplified 3 -DOF system subject to a ground motion base acceleration $\ddot{u}_{g}(t)$ read

$$
M \ddot{u}(t)+C \dot{u}(t)+K u(t)=-\tau \ddot{u}_{g}(t),
$$

with

$$
\begin{aligned}
& M=\left[\begin{array}{ccc}
1 & 1 & 0 \\
1 & 1+\mu_{b} & 0 \\
0 & 0 & \mu_{t}+\beta_{t}
\end{array}\right] \\
& C=\left[\begin{array}{ccc}
2 \zeta_{s} \omega_{s} & 0 & 0 \\
0 & 2 \zeta_{b} \omega_{b} \mu_{b}+2 \zeta_{t} \omega_{t} \mu_{\mathrm{et}} & -2 \zeta_{t} \omega_{t} \mu_{\mathrm{et}} \\
0 & -2 \zeta_{t} \omega_{t} \mu_{\mathrm{et}} & 2 \zeta_{t} \omega_{t} \mu_{\mathrm{et}}
\end{array}\right] \text {; } \\
& K=\left[\begin{array}{ccc}
\omega_{s}^{2} & 0 & 0 \\
0 & \omega_{b}^{2} \mu_{b}+\omega_{t}^{2} \mu_{\mathrm{et}} & -\omega_{t}^{2} \mu_{\mathrm{et}} \\
0 & -\omega_{t}^{2} \mu_{\mathrm{et}} & \omega_{t}^{2} \mu_{\mathrm{et}}
\end{array}\right] \\
& \tau=\left[\begin{array}{c}
1 \\
1+\mu_{b} \\
\mu_{t}
\end{array}\right] \text {; } \\
& u=\left[\begin{array}{c}
u_{\mathrm{sr}}(t) \\
u_{b}(t) \\
u_{t}(t)
\end{array}\right] \text {, }
\end{aligned}
$$

and where the following positions in terms of frequency, damping, and mass ratios of the three subsystems are introduced: 


$$
\begin{aligned}
\omega_{s} & =\sqrt{\frac{k_{s}}{m_{s}}} ; \\
\zeta_{s} & =\frac{c_{s}}{2 m_{s} \omega_{s}} ; \\
\omega_{b} & =\sqrt{\frac{k_{b}}{m_{b}}} ; \\
\zeta_{b} & =\frac{c_{b}}{2 m_{b} \omega_{b}} ; \\
\omega_{t} & =\sqrt{\frac{k_{t}}{m_{t}+b_{t}}} ; \\
\beta_{t} & =\frac{b_{t}}{m_{s}} ; \\
\mu_{t} & =\frac{m_{t}+b_{t}}{2\left(m_{t}+b_{t}\right) \omega_{t}} ; \\
\mu_{b} & =\frac{m_{b}}{m_{s}} ;
\end{aligned}
$$

wherein $b_{t}$ and $\beta_{t}$ represent the TMDI inertance and the inertance ratio, respectively, while $m_{\mathrm{et}}$ is the effective mass of the TMDI that incorporates both the physical mass $m_{t}$ and the apparent mass $b_{t}$ (i.e., related to the rotary inertia as explained above). The limit case of a base-isolated structure with attached TMD (Figure 3(a)) may be retrieved by assuming $b_{t}=0$. The inertance ratio $\beta_{t}$ multiplies the relative acceleration between points 1 and 2 , which coincides with $\ddot{u}_{t}$. We point out that while the inertial forces in the TMDI are associated with the effective mass ratio $\mu_{\text {et }}$, the earthquake-induced accelerations on the right-hand side of (3) multiply the physical mass ratio $\mu_{\mathrm{t}}$ only (see the $\tau$ vector in (4)). Therefore, due to the peculiar format of (4), increasing the inertance $\beta_{t}$ is more beneficial than increasing the TMD mass $\mu_{t}$ because it selectively raises the TMDI inertia properties without increasing the corresponding seismic force to which the device is subject. This is an extremely important consideration for limiting the TMDI stroke, as demonstrated and quantified below.

\section{Displacement Demand in Base-Isolated Structures}

In many existing structures the actual seismic joint is insufficient or nonexisting. Indeed, although modern codes such as Eurocode 8 [26] prescribe a minimum seismic separation distance between adjacent buildings, large sections of cities in seismically active regions were constructed before such requirements were introduced [5]. This problem is really important for strategic buildings in which during strong earthquake pounding damage caused to both structural and nonstructural components may have dramatic social and civil consequences. Moreover, in big and highly populated cities and metropolitan areas in which the cost of land is very high, buildings that are nearly in contact with each other, therefore prone to pounding damage, are frequent.

In order to mitigate the earthquake-induced damage to such buildings, the strategy of seismic base isolation has been widely adopted. Nevertheless, while reducing the forces in the superstructure, there is an issue when the seismic joint is quite limited, as in the cases mentioned above. Indeed, the vibration control is achieved by providing flexibility at the base of the structure such that the overall displacement of the base-isolated structure may also exceed the maximum admissible displacement resulting from the actual seismic joint. Just to fix the concepts, we suppose that the actual seismic joint is a fraction of the total height of the building $H$, for example, $H / 100$. In Figure 4, we extremely simplify the concept of displacement demand of a base-isolated structure, and we present three different situations. The first one (Figure 4(a)) concerns a typical BIS having conventional parameters of natural period and damping ratio: in this case, the BIS displacement $u_{b}$ may be higher than the admissible seismic joint, which may lead to pounding damage under a strong earthquake. In the second situation (Figure 4(b)), the BIS period is not so high, which means that the flexibility of the isolators is reduced. Although this may in turn reduce the BIS displacement $u_{b}$ so as to be lower than the seismic joint, the effectiveness of the vibration control achieved by the BIS decreases because of the reduced period, which implies higher forces and displacements transmitted to the superstructure. As a matter of fact, this seismic protection system is not convenient, and the displacements of the superstructure (e.g., the last floor displacement $u_{\text {slf }}$ ) may also exceed the seismic joint. The third and final case depicted in Figure 4(c) is related to a BIS endowed with high damping features (e.g., high-damping rubber-bearing isolators or high-performance lead rubber bearings). In this situation, the displacements of both the BIS and the superstructure are quite limited and may be lower than the seismic joint. Nevertheless, increasing the BIS damping may be detrimental for the superstructure response in terms of interstory drifts and floor accelerations, which are of great importance for nonstructural components and equipment present in the building, in addition to disturbing occupants [9]. Therefore, it seems that the conventional BIS alone is not capable of achieving an effective seismic protection and structural control of the building along with guaranteeing quite limited displacements for existing structures having limited seismic joint. As clarified below, enhancing the BIS with the supplemental rotational inertia via the concept of the TMDI could be a viable solution to this problem. 


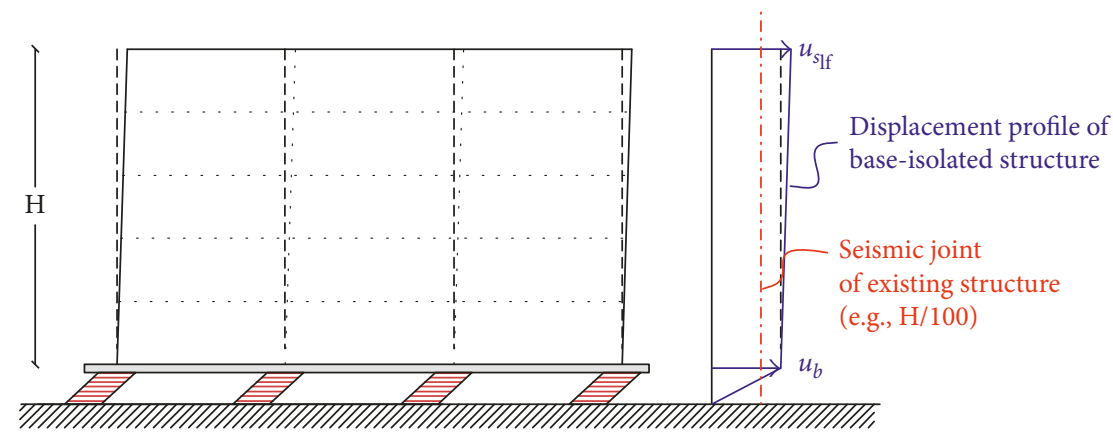

(a)

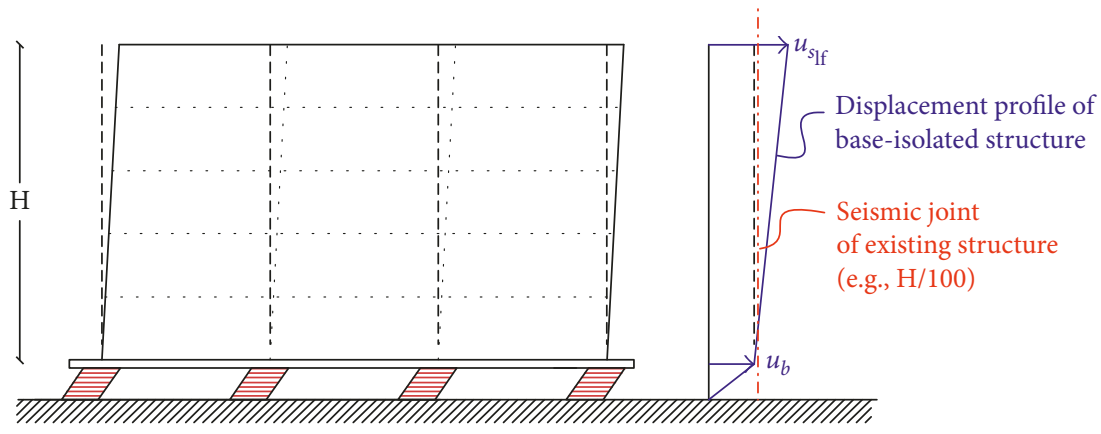

(b)

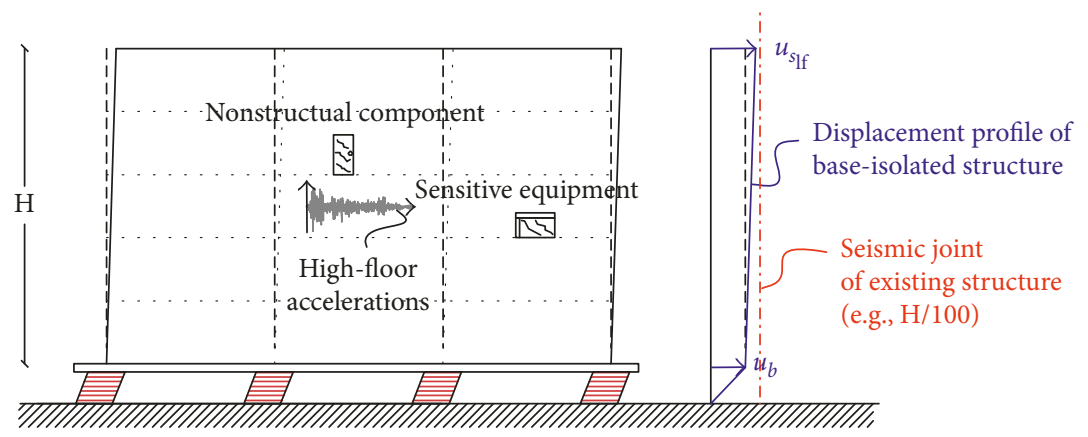

(c)

FIGURE 4: Schematic representation of the displacement demand in base-isolated structures: (a) conventional BIS with medium damping; (b) low-period BIS with medium damping; (c) high-damping BIS.

\section{Optimal Design of the TMDI and Tuning Procedure}

The selection of the BIS parameters follows the conventional rules for seismic base isolation [1]; for instance, the natural period of the BIS is assumed to be $5-10$ times higher than that of the fixed-base structure in order to achieve an effective vibration isolation. Conversely, the design of the TMDI is not straightforward as the dynamic parameters of the TMDI should be tuned such that the best vibration control is attained. An optimization problem is set up for this purpose. The optimization procedure here proposed resembles tuning procedures for TMD-based control strategies $[10,20,27,44]$, wherein some parameters are the free design variables sought in the optimization problem, while other parameters are supposed known.

The TMDI is characterized by four parameters in the most general case, namely, $\mu_{t}, \beta_{t}, \omega_{t}$, and $\zeta_{t}$. Among these four parameters, the approach pursued in this paper is to find the best frequency ratio $\nu_{t}=\omega_{t} / \omega_{b}$ and the best TMDI damping ratio $\zeta_{t}$ for fixed effective mass ratio $\mu_{\mathrm{et}}=\mu_{t}+\beta_{t}$, which is similar to other research contributions dealing with inerter-based devices in broad terms $[14,15]$. Indeed, the effective mass ratio $\mu_{\text {et }}$ can be designed according to mechanical considerations, that is, via the selection of a flywheel mass $\mu_{t}$ and an inertance ratio $\beta_{t}$ arising from (2) and Figure 1. For a given $\mu_{\text {et }}$, the optimal couple $\left(\nu_{t}, \zeta_{t}\right)$ is sought that minimizes an objective function or performance index. Such a performance index, being a representative measure of the system response, is chosen via a stochastic dynamic analysis of the system, thus accounting for the stochastic nature of the earthquake ground motion as described below.

5.1. Earthquake Input Representation. Owing to the stochastic nature of earthquake ground motion [28], the base 
TABLE 1: Filter parameters depending on soil conditions (Der Kiureghian and Neuenhofer [37]).

\begin{tabular}{lcccc}
\hline Soil type & $\omega_{g}(\mathrm{rad} / \mathrm{s})$ & $\zeta_{g}$ & $\omega_{f}(\mathrm{rad} / \mathrm{s})$ & \\
\hline Firm & 15.0 & 0.6 & 1.5 & \\
Medium & 10.0 & 0.4 & 1.0 & 0.6 \\
Soft & 5.0 & 0.2 & 0.5 & 0.6 \\
\hline
\end{tabular}

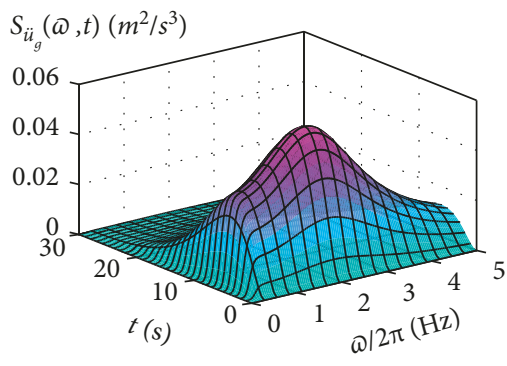

(a)

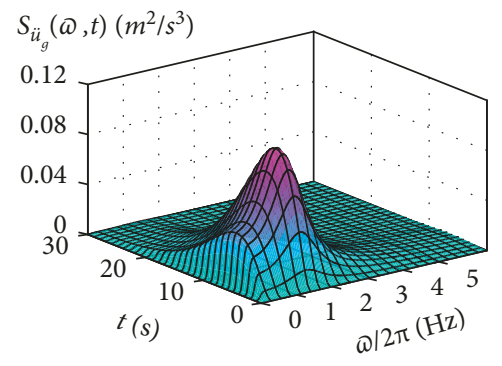

(b)

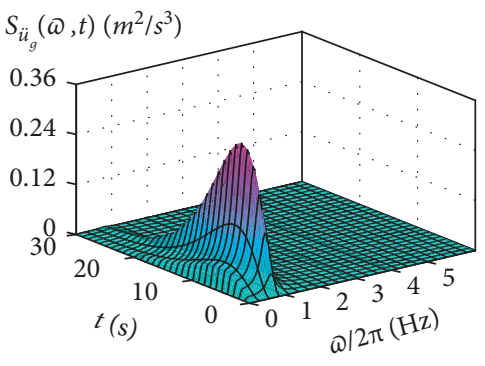

(c)

FIGURE 5: Evolutionary PSD function as per (6) for three soil conditions: (a) firm; (b) medium; (c) soft.

acceleration $\ddot{u}_{g}(t)$ entering (3) is assumed as a random process, more specifically, a quasi-stationary (uniformly modulated) Gaussian zero-mean stochastic process described by the two-sided evolutionary PSD function $[29,30]$ :

$$
S_{\ddot{u}_{g}}(\omega, t)=|\varphi(t)|^{2} S(\omega) \text {. }
$$

The time-modulating function by $\mathrm{Hsu}$ and Bernard [31] is adopted:

$$
\varphi(t)=a_{1} t \exp \left(-a_{2} t\right)
$$

in which the two constants have been set as $a_{1}=0.45 \mathrm{~s}^{-1}$ and $a_{2}=1 / 6 \mathrm{~s}^{-1}$ in accordance with statistical studies regarding the general class of accelerograms recorded during the San Fernando, California, earthquake [32].

In order to investigate the influence of soil characteristics in terms of frequency content, a filtered Gaussian white-noise process is considered for the stationary PSD function $S(\omega)$ entering (6) [33]. The widely used Kanai-Tajimi filter model $[34,35]$ is employed with a second filter in series as proposed by Clough and Penzien [32] in order to overcome the unrealistically high values of the PSD in the low-frequency regime:

$$
S(\omega)=\frac{\omega_{g}^{4}+4 \zeta_{g}^{2} \omega_{g}^{2} \omega^{2}}{\left(\omega_{g}^{2}-\omega^{2}\right)^{2}+4 \zeta_{g}^{2} \omega_{g}^{2} \omega^{2}} \frac{\omega^{4}}{\left(\omega_{f}^{2}-\omega^{2}\right)^{2}+4 \zeta_{f}^{2} \omega_{f}^{2} \omega^{2}} S_{w}
$$

where $\omega_{g}$ and $\zeta_{g}$ are the fundamental circular frequency and damping ratio of the surface soil deposits, respectively, and $\omega_{f}$ and $\zeta_{f}$ represent the additional Clough-Penzien filter parameters that control suppression of the low frequencies allowed by the Kanai-Tajimi spectrum. The $S_{w}$ parameter in (8), describing the amplitude of the bedrock excitation spectrum modelled as a white-noise process, can be related to the peak ground acceleration (PGA) $\ddot{u}_{g 0}$ according to the formula [36]:

$$
S_{w}=\frac{0.141 \zeta_{g} \ddot{u}_{g 0}^{2}}{\omega_{g} \sqrt{1+4 \zeta_{g}^{2}}}
$$

Filter parameters $\omega_{g}, \zeta_{g}, \omega_{f}$, and $\zeta_{f}$ should be appropriately selected depending on the soil condition being considered. Herein, we assume deterministic values of the filter parameters that are related to the soil stiffness according to the study by Der Kiureghian and Neuenhofer [37]. The relevant values for firm, medium, and soft soil conditions are reported in Table 1. In Figure 5, the corresponding evolutionary PSD function is plotted for the three analyzed soil conditions, by assuming a PGA equal to $\ddot{u}_{q 0}=0.3 \mathrm{~g}$. Reasonable PSD shapes result from the assumed filter parameters: for instance, the PSD for the soft soil produces a relatively narrow-band process with a predominant period of approximately $1.25 \mathrm{~s}$, which might be representative of deep alluvium sites such as parts of Mexico City, whereas the spectral shape for the firm ground is broadband and contains significant contributions from higher frequencies.

5.2. Objective Function and Numerical Algorithm. Optimum design of the novel vibration control system is based on the stochastic response of the 3 -DOF system due to the PSD function (8), thus relying on a probabilistic framework. With reference to the simplified 3-DOF system sketched in Figure 3 , a representative measure of the system response is the variance of the displacement of the structural SDOF relative to the ground, that is, of the variable $u_{s}=u_{\mathrm{sr}}+u_{b}$. Therefore, the objective function (OF) to minimize is

$$
\mathrm{OF}=\sigma_{u_{s}}^{2}=E\left[u_{s}^{2}\right]=\sigma_{u_{\mathrm{sr}}}^{2}+\sigma_{u_{b}}^{2}+2 \sigma_{u_{\mathrm{sr}} u_{b}} .
$$

It will be demonstrated that minimizing $\sigma_{u}^{2}$ has a twofold effect, namely, reducing the displacement in the BIS $u_{b}$ and lowering the relative displacement between the superstructure and the BIS $u_{\mathrm{sr}}$. 


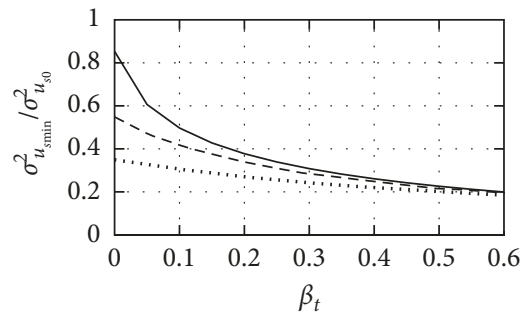

Firm soil conditions

$$
\begin{aligned}
& -\mu_{t}=0.01 \\
& ---\mu_{t}=0.1 \\
& \ldots \ldots \mu_{t}=0.4
\end{aligned}
$$

(a)

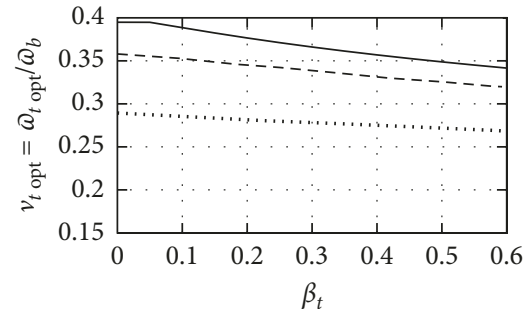

Firm soil conditions

$$
\begin{aligned}
& -\mu_{t}=0.01 \\
& ---\mu_{t}=0.1 \\
& \ldots \ldots \mu_{t}=0.4
\end{aligned}
$$

(b)

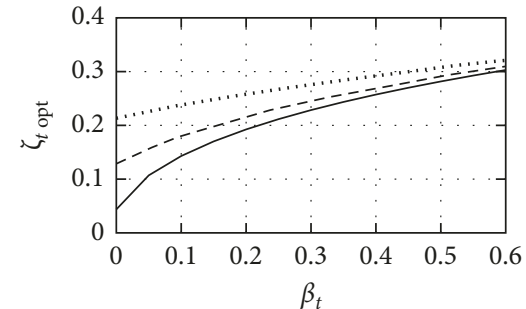

Firm soil conditions

$-\mu_{t}=0.01$

- - $\mu_{t}=0.1$

..... $\mu_{t}=0.4$

(c)

FIgURE 6: Optimal TMDI tuning for firm soil conditions: (a) minimum OF achieved, normalized with respect to the case without TMDI; (b) optimal frequency ratio; (c) optimal damping ratio.

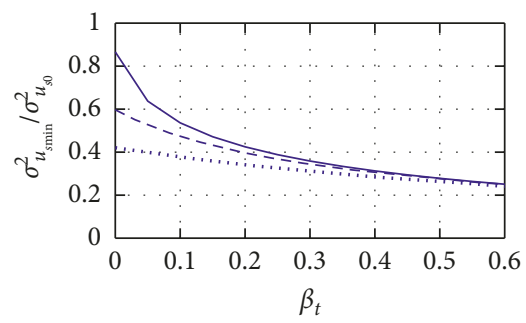

Medium soil conditions

$$
\begin{aligned}
-\mu_{t} & =0.01 \\
---\mu_{t} & =0.1 \\
\ldots \ldots & \mu_{t}=0.4
\end{aligned}
$$

(a)

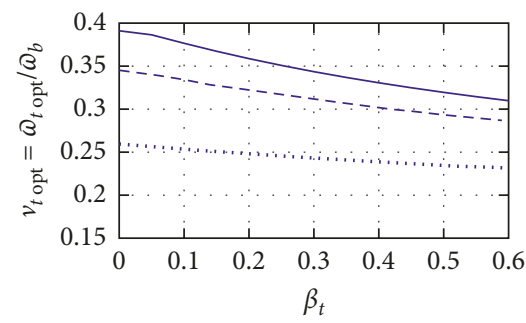

Medium soil conditions

$-\mu_{t}=0.01$

$--\mu_{t}=0.1$

…... $\mu_{t}=0.4$

(b)

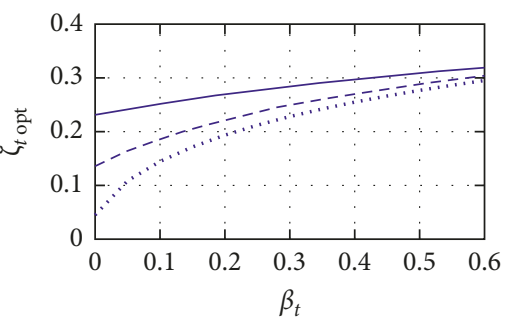

Medium soil conditions

$$
\begin{aligned}
-\mu_{t} & =0.01 \\
---\mu_{t} & =0.1 \\
\ldots \ldots & \mu_{t}=0.4
\end{aligned}
$$

(c)

FIGURE 7: Optimal TMDI tuning for medium soil conditions: (a) minimum OF achieved, normalized with respect to the case without TMDI; (b) optimal frequency ratio; (c) optimal damping ratio.

The optimization problem dealt with in this paper is handled via a numerical search algorithm, through the builtin MATLAB fmincon function, which is a nonlinear program solver (adopting the interior-point algorithm) with embedded constraint functions for the sought TMDI tuning variables $x=\left[v_{t}, \zeta_{t}\right]$. The lower bound and upper bound vectors are assumed as (in MATLAB vector notation) $x_{\mathrm{lb}}=$ $[0.10,0.01]$ and $x_{\mathrm{ub}}=[2.00,1.00]$. The tuning variables $v_{t}$ and $\zeta_{t}$ span their allowed ranges with an increment of $10^{-7}$ and the tolerance on the achieved minimum OF is $10^{-7}$. In mathematical terms, the following nonlinear constrained single-objective multivariable optimization problem is to be solved:

$$
\min _{x} \sigma_{u_{s}}^{2}(x) \text { such that } x_{\mathrm{lb}} \leq x \leq x_{\mathrm{ub}} .
$$

5.3. Parametric Study. The optimal design of the TMDI to achieve a reduction of the displacement demand in a baseisolated structure is discussed here. A wide parametric study is carried out, wherein the main investigated parameters are (1) the soil condition (firm, medium, soft type, and the white-noise process as an extremely broadband frequency content); (2) the physical mass ratio $\mu_{t}$; (3) the inertance (or apparent mass) ratio $\beta_{t}$; and (4) the BIS damping ratio $\zeta_{b}$. The damping ratio of the structural SDOF is assumed as $\zeta_{s}=0.02$, which is a rather typical value if limited damage is expected in the superstructure. Moreover, an isolation ratio in terms of frequency ratio $v_{b}=\omega_{b} / \omega_{s}=0.2$ and a mass ratio $\mu_{b}=0.2$ have been assumed throughout.

In Figures 6-9, the optimal TMDI parameters are shown for firm, medium, soft soil conditions, and for a white-noise (broadband) earthquake input. A value of $\zeta_{b}=0.2$ is here assumed, as the influence of $\zeta_{b}$ is analyzed later. For practical purposes, the minimum achieved $\sigma_{u_{s}}^{2}$ value is shown normalized with respect to the case without TMDI, that is, the displacement variance of the structural SDOF in a conventional base isolation scheme $\sigma_{u_{s 0}}^{2}$. As a result, values of the $\sigma_{u_{\mathrm{s}}}^{2} / \sigma_{u_{\mathrm{s}}}^{2}$ ratio lower than the unity means that the proposed control strategy is effective in the displacement reduction, with $\left(1-\sigma_{u_{s}}^{2} / \sigma_{u_{s}}^{2}\right) \times 100$ being a percentage measure of such reduction. The design graphs also include the limit case of the TMD in place of the TMDI for a zero value of the inertance ratio, that is, $\beta_{t}=0$. Three different values of the $\mu_{t}$ 


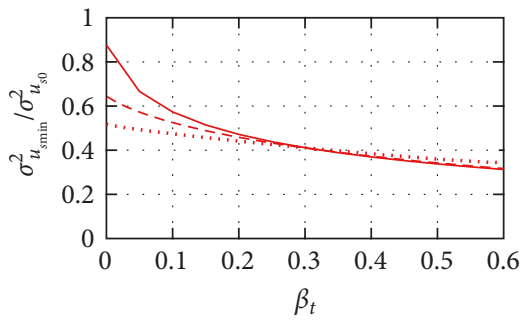

Soft soil conditions

$-\mu_{t}=0.01$

$---\mu_{t}=0.1$

$\ldots \ldots \mu_{t}=0.4$

(a)

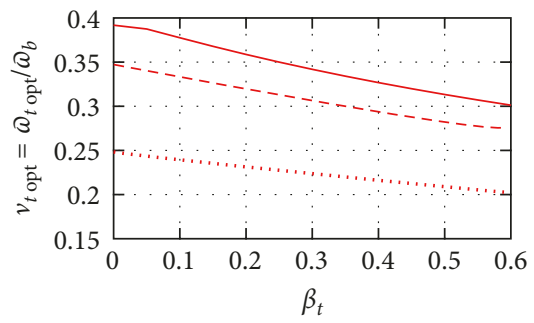

Soft soil conditions

$$
\begin{aligned}
-\mu_{t} & =0.01 \\
---\mu_{t} & =0.1
\end{aligned}
$$$$
\ldots \ldots \mu_{t}=0.4
$$

(b)

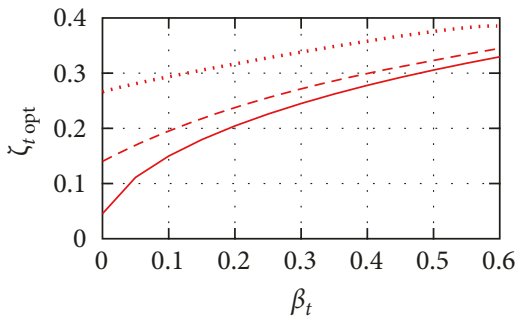

Soft soil conditions

$-\mu_{t}=0.01$

$---\mu_{t}=0.1$

$\ldots \ldots \mu_{t}=0.4$

(c)

FIgURE 8: Optimal TMDI tuning for soft soil conditions: (a) minimum OF achieved, normalized with respect to the case without TMDI; (b) optimal frequency ratio; (c) optimal damping ratio.

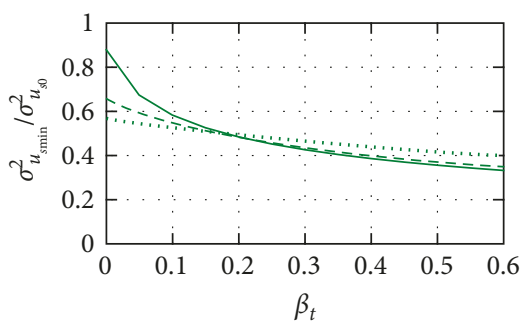

White-noise assumption (extremely broadband frequency content)

$$
\begin{aligned}
-\mu_{t} & =0.01 \\
---\mu_{t} & =0.1 \\
\ldots \ldots & \mu_{t}=0.4
\end{aligned}
$$

(a)

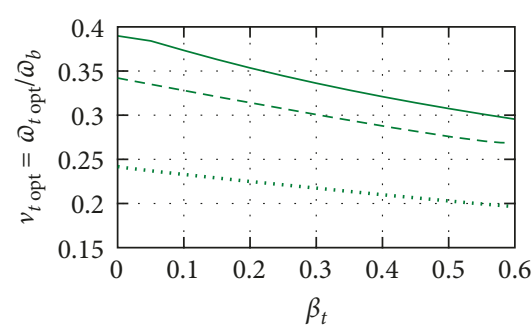

White-noise assumption (extremely broadband frequency content)

$$
\begin{aligned}
-\mu_{t} & =0.01 \\
---\mu_{t} & =0.1 \\
\ldots \ldots & \mu_{t}=0.4
\end{aligned}
$$

(b)

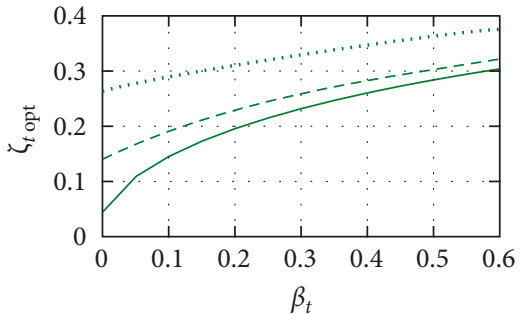

White-noise assumption (extremely broadband frequency content)

$$
\begin{aligned}
-\mu_{t} & =0.01 \\
---\mu_{t} & =0.1 \\
\ldots \ldots, \mu_{t} & =0.4
\end{aligned}
$$

(c)

FIGURE 9: Optimal TMDI tuning for white-noise assumption for the earthquake: (a) minimum OF achieved, normalized with respect to the case without TMDI; (b) optimal frequency ratio; (c) optimal damping ratio.

ratio are shown, namely $\mu_{t}=0.01$ that is representative of a small-mass flywheel device as shown in Figure $2, \mu_{t}=0.1$ that is relevant to a conventional TMD system, and $\mu_{t}=0.4$ representing a nonconventional TMD that exploits masses already present in the system $[15,38]$.

By inspection of the OF achieved, the TMDI appears to be very effective in the displacement reduction of the baseisolated structure. In particular, depending on the soil conditions, a $60-70 \%$ reduction of the displacement variance is achieved with an inertance ratio $\beta_{t}=0.4$, whereas the displacement reduction attained by a nonconventional TMD having $\mu_{t}=0.4$ is slightly lower (cf. the values at the intersection with the vertical axis, limit case $\beta_{t}=0$ ). However, we stress that high values of the inertance ratio $\beta_{t}$ can be accomplished without increasing the inerter's physical mass, but rather via the adjustment of the gearing ratios (or simply by setting two or more flywheels in series [16]). This means that high vibration control performance can be achieved by the TMDI without resorting to excessively large secondary mass, as implied in the nonconventional TMD. In other words, the TMDI outperforms the classical TMD as it exploits the light-weight features of the conventional TMD, with the effectiveness of the nonconventional TMD [15].

The optimal TMDI parameters $\nu_{t \text { opt }}$ and $\zeta_{t \text { opt }}$ are significantly affected by the physical mass $\mu_{t}$ and by the inertance ratio $\beta_{t}$. Generally speaking, for all the soil conditions, $v_{t \text { opt }}$ decreases with increasing $\mu_{t}$ and with increasing $\beta_{t}$. Conversely, the optimal damping ratio $\zeta_{t \text { opt }}$ follows an opposite different trend, that is, it increases with both increasing physical mass and/or apparent mass. The influence of the soil condition is considerable: the soil stiffness leads to higher frequency ratios $v_{t \text { opt }}$ and lower damping ratios $\zeta_{t \text { opt }}$, and this variability turns out to be more important when high values of $\mu_{t}$ or $\beta_{t}$ are considered. Interestingly, the optimal parameters found under the white-noise assumption are nearly similar to those obtained for soft soil conditions. Finally, the vibration control performance of the system reduces when passing from firm to soft soil conditions; this is consistent with the expectations, as the soft soil condition leads to a predominance of longperiod components (such as that obtained in Mexico City). The assembled base-isolated system is rather vulnerable to 


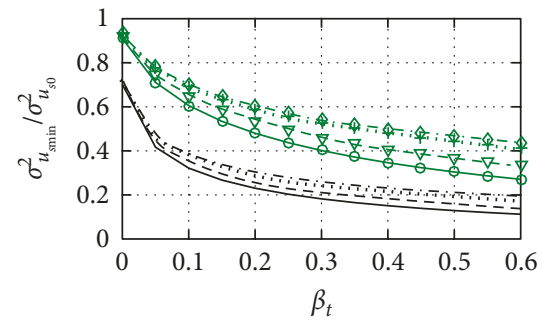

$$
\begin{array}{ll}
\zeta_{b}=0.1(\mathrm{LDRB}) & \zeta_{b}=0.3(\mathrm{LDRB}) \\
- \text { Firm } & \multimap-\text { Firm } \\
--- \text { Medium } & -\nabla-\text { Medium } \\
\ldots \ldots \text { Soft } & \ldots+\cdots \text { Soft } \\
\text {.-.- White-noise } & . \diamond-\text { White-noise }
\end{array}
$$

(a)

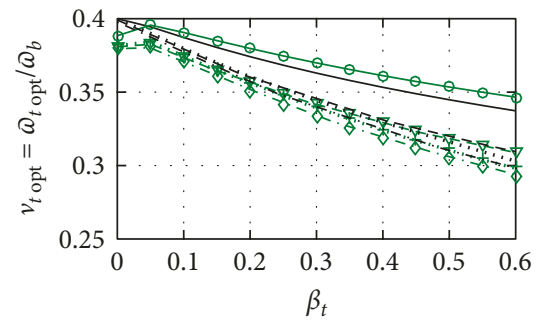

$$
\begin{array}{ll}
\zeta_{b}=0.1(\mathrm{LDRB}) & \zeta_{b}=0.3(\mathrm{LDRB}) \\
- \text { - Firm } & \multimap \text { - Firm } \\
--- \text { Medium } & -\nabla-\text { Medium } \\
\ldots . . . \text { Soft } & \text {. }+\cdot \text { Soft } \\
\text {-.-- White-noise } & . \diamond-\text { White-noise }
\end{array}
$$

(b)

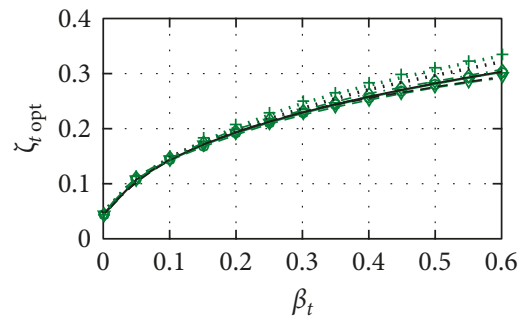

$$
\begin{aligned}
& \zeta_{b}=0.1(\mathrm{LDRB}) \quad \zeta_{b}=0.3(\mathrm{LDRB}) \\
& \text { - Firm } \rightarrow \text { Firm } \\
& \text {--- Medium - -5-Medium } \\
& \text {..... Soft } \quad \text {..'. Soft } \\
& \text {.-.- White-noise . } \diamond-\text { White-noise }
\end{aligned}
$$

(c)

FIGURE 10: Optimal TMDI design for different BIS damping ratios and soil conditions: (a) minimum OF achieved, normalized with respect to the case without TMDI; (b) optimal frequency ratio; (c) damping ratio.

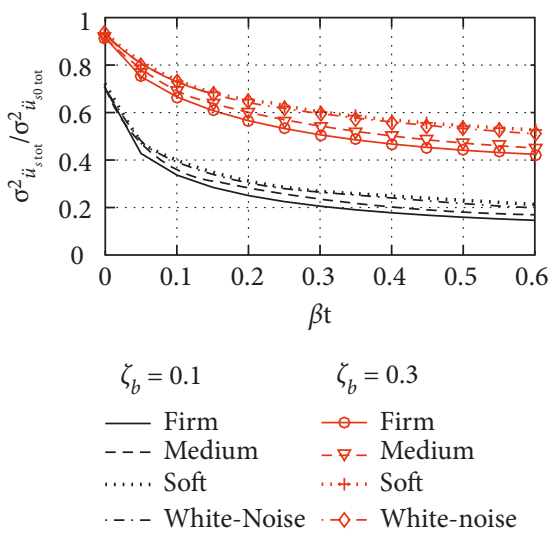

(a)

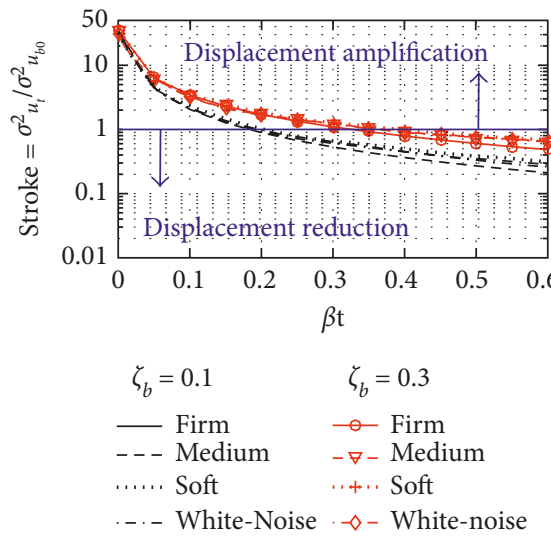

(b)

Figure 11: Optimal TMDI design for different BIS damping ratios and soil conditions: (a) normalized total acceleration variance and (b) normalized TMDI displacement variance, representative of the TMDI stroke.

earthquake excitations whose frequency content is concentrated in the long-period range [6].

The influence of the BIS damping ratio $\zeta_{b}$ is investigated in Figures 10 and 11. Two $\zeta_{b}$ values are analyzed, namely, $\zeta_{b}=0.1$, representative of low-damping rubber bearings (LDRBs), and $\zeta_{b}=0.3$, typical of high-damping rubber bearing (HDRB) as well as of lead rubber bearings (LRBs). In accordance with other literature findings related to TMDbased systems [18], it is found that the effectiveness of the TMDI increases when low-damping isolation bearings are addressed (cf. Figure 10(a)). For instance, adopting $\beta_{t}=0.2$ the reduction of the displacement variance is more than doubled in passing from $\zeta_{b}=0.3$ to $\zeta_{b}=0.1$, regardless of the soil condition (cf. Figure 10(a)).

Furthermore, this reduction is not confined to the displacement variance. In Figure 11, we also report the superstructure total acceleration variance, computed as $\sigma_{\ddot{u}_{s t o t}}^{2}=E\left[\ddot{u}_{s \text { tot }}^{2}\right]=4 \zeta_{s}^{2} \omega_{s}^{2} \sigma_{\dot{u}_{\mathrm{sr}}}^{2}+\omega_{s}^{4} \sigma_{u_{\mathrm{sr}}}^{2}$, and the variance of the TMDI displacement $\sigma_{u_{t}}^{2}$ characterizing the so-called TMDI stroke. Normalization of these quantities is again performed.
The TMDI displacement variance, shown in a semilog plot for improved readability, is divided by the BIS displacement variance in the uncontrolled case $\sigma_{u_{b 0}}^{2}$. To make the results more evident, a thicker solid horizontal line is drawn at a unitary value of the normalized TMDI stroke to distinguish between amplification and reduction as compared to the conventional base isolation scheme.

It can be noted that the reduction of the superstructure acceleration is improved when low-damping isolators are employed (Figure 11(a)). Similar effects are observed with regard to the TMDI stroke: the displacement of the secondary mass is more effectively controlled for low-damping BIS because the range of displacement reduction widens when lowering the $\zeta_{b}$ value, and the attained $\sigma_{u_{t}}^{2}$ value decreases accordingly (Figure 11(b)). It seems that the inertance ratio should be set such that $\beta_{t}>0.3$ in order to achieve an effective vibration control of the base-isolated structure (in terms of displacements and accelerations) without implying a large TMDI stroke. Looking at the obtained graphs, $\beta_{t}=0.4$ seems to be a reasonable inertance 
ratio for design purposes. All the aspects discussed in this parametric study are of extreme importance for a practical implementation of this system into existing structures, as will be clarified in the following sections.

\section{Implementation of the Proposed System into Existing Structures}

The optimal tuning of the system discussed in Section 5 is the starting point for a practical implementation of the proposed system into existing structures, which is the main goal of this research work. The analysis is here extended to multidegree-of-freedom (MDOF) systems like multistory buildings. To this aim, the superstructure DOF is meant as the fundamental mode of vibration of the superstructure. More importantly, a possible strategy of implementation into existing structures comprising an additional set of isolators to attach the inerter to the structure is presented here. The aspects discussed in Section 5.3 are briefly summarized here. It has been found that the for firm soil conditions, the optimal TMDI frequency should be higher than that for the soft soil conditions. On the contrary, it seems that the optimal TMDI damping ratio is lower for the firm soil conditions than that for the soft soil conditions. The influence of the soil condition on the TMDI optimal design is more pronounced for high values of $\mu_{t}$ or $\beta_{t}$. Moreover, an inertance ratio at least $\beta_{t}=0.35-0.4$ is essential for keeping the TMDI stroke limited.

For a possible implementation strategy of this system into existing structures, we now approach the problem of designing the base isolation system from a different perspective. The elastic and damping properties of the TMDI system, $k_{t}$ and $c_{t}$, may be meant as the effective stiffness and equivalent viscous damping ratio of an auxiliary set of isolators acting in parallel with the conventional isolators; the latter is featured by corresponding $k_{b}$ and $c_{b}$, as sketched in Figure 12. The only difference between these two groups of isolators is that the auxiliary isolators are equipped with an inerter device, which connects them to the ground. Therefore, this model idealization represents an enhanced base-isolation system with a particular arrangement of conventional isolators, auxiliary isolators, and inerter. This schematic arrangement is a possible implementation strategy of the proposed system into existing structures. In this way, the inerter can easily be connected to the structure via the auxiliary isolators and a sliding device. The mass $m_{t}$ comprises the flywheel mass and the mass of the rigid element that connects the auxiliary isolators with each other, for example, a basement subunit. On the basis of the optimal TMDI parameters found in Section 5.3, the auxiliary isolators should be more flexible $\left(\omega_{t \text { opt }} \approx 0.35 \omega_{b}\right)$ and should have more inherent damping $\left(\zeta_{\text {topt }} \approx 0.2-0.3\right)$ than the conventional isolators, in order for the proposed system to perform at its best. Based on these considerations, a new strategy of seismic retrofitting of existing structures is proposed in this paper that consists in a combination of low-damping conventional isolators, for example, $\zeta_{b} \approx 0.1$, in parallel with medium-to-high damping flexible auxiliary isolators, for

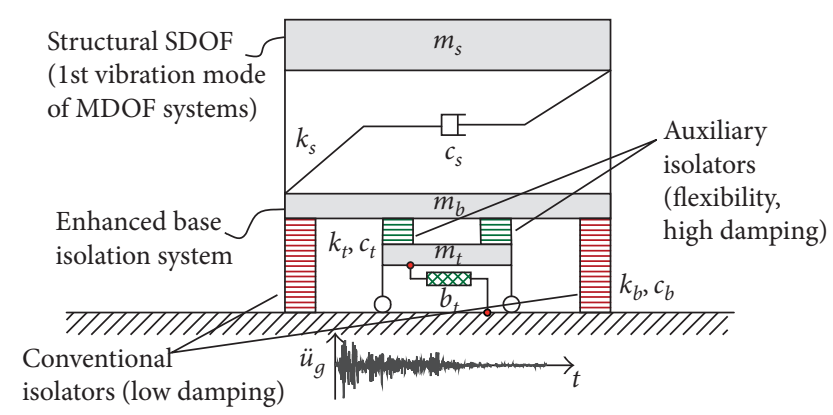

FIGURE 12: Schematic arrangement of the proposed base isolation system comprising two sets of isolators to attach the inerter to the structure.

example, $\zeta_{t} \approx 0.1$, the latter installed in conjunction with an inerter device having inertance ratio around $\beta_{t}=0.4$.

To summarize the basic steps of a schematic seismic retrofitting procedure via the proposed system discussed above, in Figure 13 a conceptual flowchart is elaborated. Obviously, this flowchart is an extremely simplified idealization of the several complex design stages for a real seismic retrofitting procedure. A series of bullet points highlight the main procedural steps in a schematic way. Once a given existing structure is characterized in terms of dynamic properties, soil conditions, and importance factor, the design of the retrofitting system is normally based on constraints such as a limited seismic joint or the presence of adjacent buildings. First, the set of conventional isolators is designed. As a second step, the properties of the inerter device are chosen on the basis of an expected value of the performance index, which is in turn related to the desired level of vibration reduction to achieve. For given properties of the inerter device in terms of mass and rotational inertia (inertance), the set of auxiliary isolators are designed on the basis of the design graphs discussed in Section 5.3. An overall check of the entire system should be made via timehistory linear or nonlinear analyses employing design earthquakes that are relevant to the installation site, soil conditions, and importance factor of the building. The verification is made with respect to both displacements and stress in the superstructure. Unsatisfactory displacement reduction (e.g., it is found that the displacements still exceed the seismic joint) may be faced via increasing the inertance properties (mass and rotational inertia), whereas the presence of high stresses in the superstructure (e.g., bending moments, tensile forces, floor accelerations for sensitive equipment, and so forth) can be avoided through a global amendment of the base isolation system as a whole, including the dynamic characteristics of conventional isolators and the resulting properties of auxiliary isolators and the inerter.

\section{Base Isolation with Supplemental Damping versus Supplemental Rotational Inertia}

In line with the conceptual flowchart introduced in Figure 13 , in this section, we check the effectiveness of the proposed seismic retrofitting procedure via time-history analyses. Two 


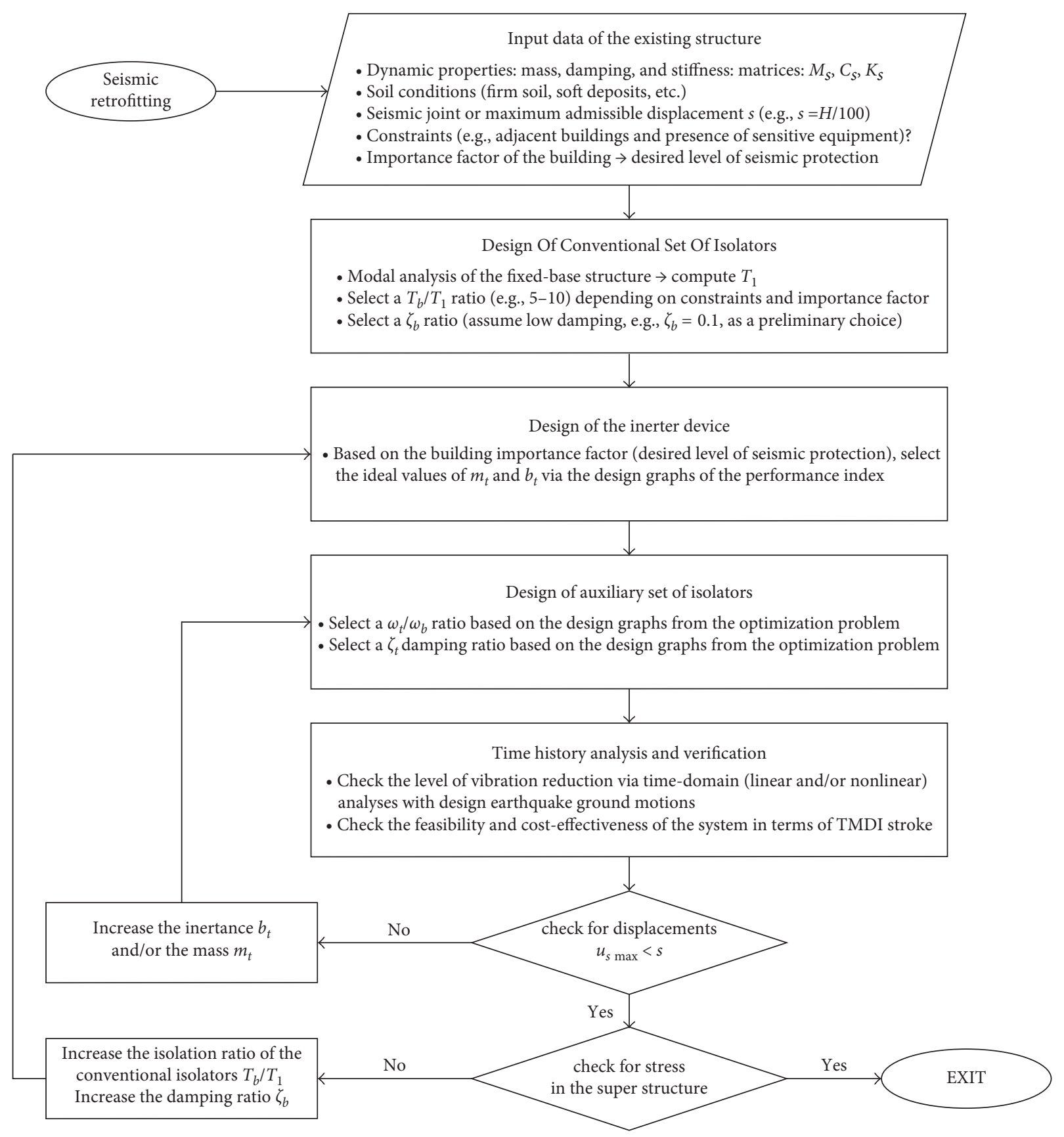

Figure 13: Conceptual flowchart of a seismic retrofitting procedure based on the proposed system of Figure 12.

different strategies are analyzed and compared, namely, the seismic base isolation with supplemental damping, and the seismic base isolation with supplemental rotational inertia by means of the TMDI. A third vibration control system combining the seismic base isolation with an attached TMD is also discussed as the limit case of the TMDI for a zero value of inertance, that is, by setting $b_{t}=0$.

7.1. Numerical Application. The existing structure is a fivestory reinforced concrete (RC) building. For simplicity, we assume that the building is symmetric in plan, so that it can be analyzed through the equivalent planar frame depicted in Figure 14. Obviously, un-symmetric plan buildings are more realistically found as existing structures, which requires a $3 \mathrm{D}$ model instead of a planar frame. However the aim of this paper is just to present and compare different seismic retrofitting strategies; therefore, a quite simple structure and a planar model are not expected to majorly prejudice the generality of the discussion.

According to Eurocode 8 requirements [39], information for structural assessment should be collected from original outline construction drawings, survey, in situ inspection, in situ testing via destructive- and/or nondestructive tests, and so forth. Geometric data regarding cross-sectional properties of beam and column elements 


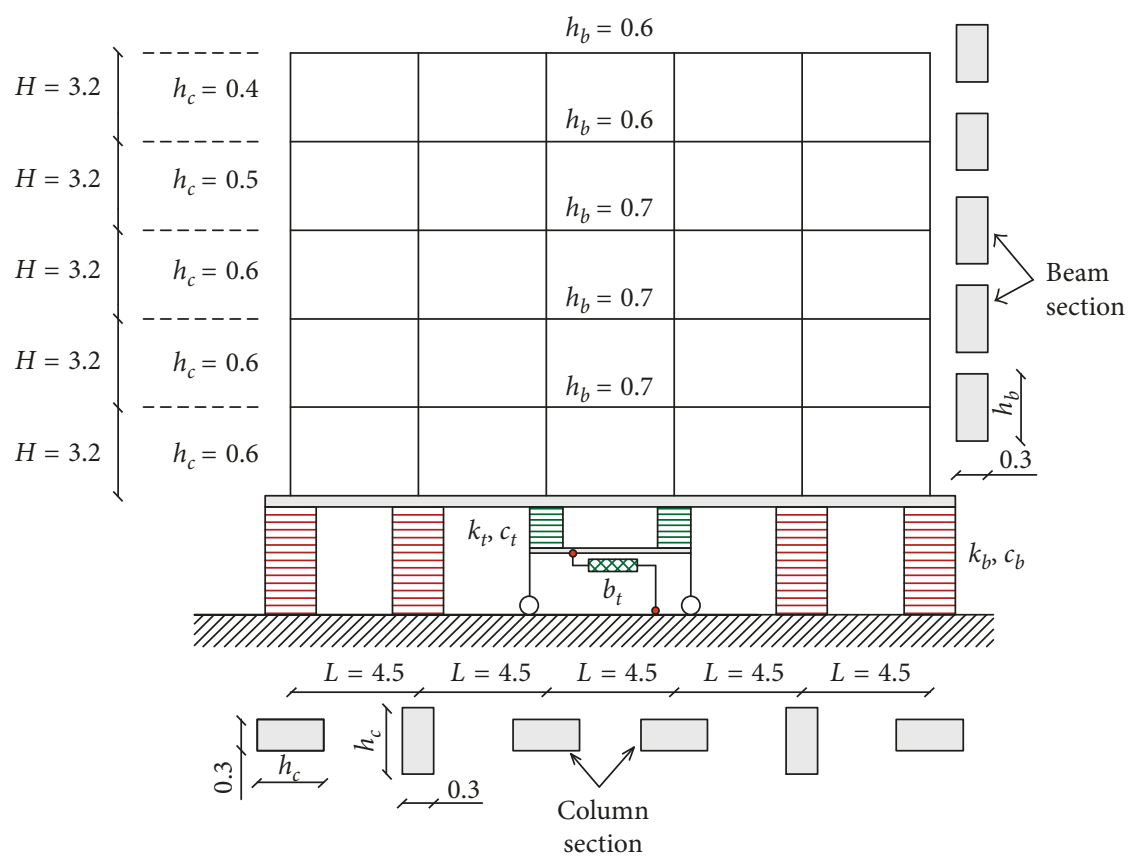

FIGURE 14: Five-story RC building with the seismic protection system proposed in this paper (all dimensions in m).

are reported in Figure 14. We assume that bay width $L$ and column height $H$ are constant at every story, with $L=4.5 \mathrm{~m}$ and $H=3.2 \mathrm{~m}$. The elastic modulus of the material is $E=$ $26,000 \mathrm{MN} / \mathrm{m}^{2}$ [40]. The second-moment of the crosssectional area is computed according to the column and beam sections reported in Figure 14. A static condensation method is applied in order to eliminate the (zero-mass) rotational DOFs [41]. With axial deformations in beams and columns neglected, the frame has 35 DOFs, with 5 dynamic DOFs represented by the displacements of the 5 stories relative to the BIS. The mass of the superstructure is thus lumped at the floor level, with $m_{s j}=60,000 \mathrm{~kg}$ denoting the mass for the first 4 floors $(j=1, \ldots, 4)$, and $m_{s 5}=50,000 \mathrm{~kg}$ for the fifth floor. Considering the importance factor of the building, the actual seismic joint, and the presence of nonstructural equipment, the following constraints should be met:

maximum displacement (or drift index) $u_{s \max }<s$ with $s=\frac{H}{100}=0.16 \mathrm{~m}$,

$$
\begin{aligned}
& \text { maximum interstory drift index } \frac{\Delta h}{H} \\
& =0.30 \% \longrightarrow \Delta h<0.0096 \mathrm{~m},
\end{aligned}
$$

maximum floor acceleration $\ddot{u}_{s \max }<0.2 \mathrm{~g}$.

In a seismic retrofitting procedure, the description of the seismic motion may be made by using artificial accelerograms and recorded or simulated accelerograms [25] that are consistent with the soil characteristics of the installation site. In this paper, artificial samples are generated in accordance with the evolutionary PSD function defined in (6) by means of the superposition of $N$ harmonic waves through the spectral representation method [42]:

$\ddot{u}_{g}^{(r)}(t)=\varphi(t) \sqrt{2} \sum_{i=0}^{N-1} \sqrt{2 S(i \Delta \omega) \Delta \omega} \cos \left(i \Delta \omega t+\phi_{i}^{(r)}\right)$,

where $\phi_{i}^{(r)}$ are the independent random phases uniformly distributed over the interval $[0,2 \pi], \Delta \omega=\omega_{c} / N$ with $\omega_{c}$ being the upper cutoff frequency beyond which $S(\omega) \approx 0$.

We assume that the building is installed in firm soil conditions. According to the Monte Carlo method, an ensemble of 100 artificial uniformly modulated accelerograms is generated from the firm-soil evolutionary PSD function presented in Section 5.1 (time duration of $30 \mathrm{~s}$ and PGA equal to $\ddot{u}_{g 0}=0.2 \mathrm{~g}$ are assumed). Time-history analyses are carried out via direct integration of the equations of motion, and the relevant statistics of the structural response are evaluated by averaging the results.

Following the conceptual flowchart of Figure 13, once the input data of the existing structure are established, the modal analysis of the fixed-base structure is carried out, which leads to the determination of the natural periods $T_{s i}[\mathrm{~s}]=[0.501,0.189,0.112,0.079,0.056]$ and the participating mass ratios $\epsilon_{s i}[\%]=[81.77,10.67,3.96,2.55,1.04]$. From Figure 14, the base isolation system relevant to the planar frame under study is composed of an ensemble of 6 isolators, two of which (the so-called auxiliary isolators) are smaller in size (i.e., more flexible) and equipped with rotational inertia via an inerter device. The basement mass is set as $m_{b}=50,000 \mathrm{~kg}$, hence resulting in a mass ratio $\mu_{b} \simeq 0.17$. As a preliminary choice, we assume an effective period of the conventional isolators $T_{\text {eff }}=2 \mathrm{~s}$ and an 
TABLE 2: Seismic retrofitting strategies analyzed and compared in this study and optimal parameters.

\begin{tabular}{|c|c|c|c|c|c|}
\hline Seismic retrofitting label & Seismic protection strategy & $\zeta_{b}$ & $T_{\text {eff }}(\mathrm{s})$ & $\zeta_{t}$ & $\omega_{t}(\mathrm{rad} / \mathrm{s})$ \\
\hline$\# 1$ & Conventional BIS & 0.1 & 2.0 & - & - \\
\hline$\# 2$ & BIS with rotational inertia (TMDI) & 0.1 & 2.0 & 0.256 & 2.502 \\
\hline$\# 3$ & BIS with TMD & 0.1 & 2.0 & 0.135 & 2.713 \\
\hline$\# 4$ & BIS with supplemental damping & 0.3 & 2.0 & - & - \\
\hline$\# 5$ & BIS with supplemental damping & 0.4 & 2.0 & - & - \\
\hline
\end{tabular}

equivalent viscous damping ratio $\zeta_{b}=0.1$, which implies the use of low-damping rubber bearings in line with the previous remarks. Note that $T_{\text {eff }}$ is related to $\omega_{b}$ via the expression $T_{\text {eff }}=T_{b} \sqrt{1+\mu_{b}^{-1}}$ with $T_{b}=2 \pi / \omega_{b}$, which is due to the definition of $\omega_{b}$ made in (5). Based on the design graphs developed in Section 5.3, the inerter is designed such that $\beta_{t}=0.4$ and $\mu_{t}=0.01$. Considering the total mass of the superstructure, this implies that $m_{t}=2900 \mathrm{~kg}$ and $b_{t}=116,000 \mathrm{~kg}$. The damping coefficients of the superstructure are assumed to be stiffness-proportional ("classically damped system") with $\zeta_{s i}=0.02$ with $i=1, \ldots, 5$. All these data serve to detect the optimal frequency and damping ratio of the TMDI via the design graphs developed in Section 5.3. The following optimal parameters are found: $\omega_{\text {topt }}=2.502 \mathrm{rad} / \mathrm{s}$ and $\zeta_{t \text { opt }}=0.256$. For comparison purposes, the base isolation system with attached TMD is also studied by assuming $\beta_{t}=0$ and $\mu_{t}=0.1$, and the optimal parameters found are $\omega_{t \text { opt }}=2.713 \mathrm{rad} / \mathrm{s}$ and $\zeta_{\text {topt }}=0.135$. Alternative seismic retrofitting strategies investigated below are the conventional base isolation system (without TMDI system) equipped with supplemental damping instead of rotational inertia. To this aim, we have considered increasing values of the BIS damping ratio, namely, $\zeta_{b}=0.3$ and $\zeta_{b}=0.4$. Overall, five different seismic retrofitting strategies are analyzed as summarized in Table 2.

The results from 100 time-history analyses (each relevant to a given accelerograms) are averaged to obtain the mean root-mean-square (RMS) values and the mean maximum (MAX) values of a few representative response indicators that are reported in Tables 3 and 4, respectively. A selection of response indicators that are useful and of practical importance for design engineers is computed for the five retrofitting systems, including the last-floor displacement $u_{s 5}$, the second interstory drift $\Delta u_{s 2}=u_{s 2}-u_{s 1}$, the fourth floor acceleration $\ddot{u}_{s 4}$, the base shear $V_{b}=\sum_{i} \omega_{s i}^{2} \gamma_{s i}^{2} d_{s i}$, the superstructure kinetic energy $T_{s}=1 / 2 \dot{u}_{s}^{T} M_{s} \dot{u}_{s}$, the superstructure elastic strain energy $E_{s}=1 / 2 u_{\mathrm{sr}}^{T} K_{s} u_{\text {sr }}$, the BIS displacement $u_{b}$, and the TMDI/TMD stroke $u_{t}$. In this way, an overall assessment of the performance of each seismic retrofitting strategy is made for a consistent comparison. Percentage of reductions achieved as compared to the conventional base isolation scheme (reference solution) is reported in brackets: a negative value indicates reduction and a positive one means amplification. By inspection of the Table 3 and Table 4, it is found that the best seismic retrofitting strategy is the BIS with supplemental rotational inertia (TMDI) that achieves an excellent level of vibration reduction, not only in terms of displacement demand of the base isolation system (with reductions of almost 55\% for the
RMS values and of $43 \%$ for the MAX values) but also with regard to all the other response indicators of the superstructure. For example, the kinetic energy is brought down of about $75 \%$ and $60 \%$ when the RMS and MAX values are considered, respectively. Similar considerations may be drawn for the base shear, with reductions of $53 \%$ and $38 \%$.

Such performance of the system \#2 is achieved without requiring large physical mass and without implying excessively large TMDI strokes: indeed, the displacements of the auxiliary isolators are reduced of around 26\% and 15\% (RMS and MAX values, resp.) as compared to the displacement of the conventional BIS in the uncontrolled case $u_{b 0}$, which makes the proposed system very convenient for practical applications. Conversely, if the BIS is combined with a TMD (system \#3), the vibration reductions of all the response indicators are considerably lower than those of the previous case with the TMDI. Additionally, the vibration control is accomplished at the expense of very large TMD strokes, with values of $u_{t}$ even higher (not lower) than $100 \%$ in comparison with the BIS displacement $u_{b 0}$ of the uncontrolled case (maximum displacement achieved is $48 \mathrm{~cm}$ ). This means that a large space in the building should be devoted to the TMD in order to accommodate the displacement demand of this secondary mass. This makes the difference between a passive vibration control system equipped with large mass and a one endowed with large rotational inertia (inertance), with the latter selectively raising the inertia properties without amplifying the corresponding seismic force to which the device is subject, but rather suppressing the level of ground shaking. Additionally, it is reasonably expected that using an inerter with large inertance ratios is by far cheaper than employing a TMD with very large mass amounts. This aspect may have important implications in the potential construction costs of these two alternative systems. Finally, the analysis of the results achieved by the systems \#4 and \#5, namely, the BIS with supplemental damping, reveals a poorer performance than system \#2 in terms of RMS and MAX values and with regard to all the analyzed response indicators despite the very high values of damping ratios adopted. For instance, the second interstory drift is reduced to around $40 \%$ and $28 \%$ (RMS and MAX values, resp.) as compared to the conventional BIS, while system \#2 with rotational inertia attains a reduction of $52 \%$ and $37 \%$.

In Figures 15(a) and 15(b), two arbitrary time histories of the last-floor displacement response (picked out from the 100 time-history responses for the 50th and 100th ground motion of the ensemble) are illustrated. These plots reveal that in order for the TMD/TMDI to be fully effective, the seismic input energy should penetrate into the system 


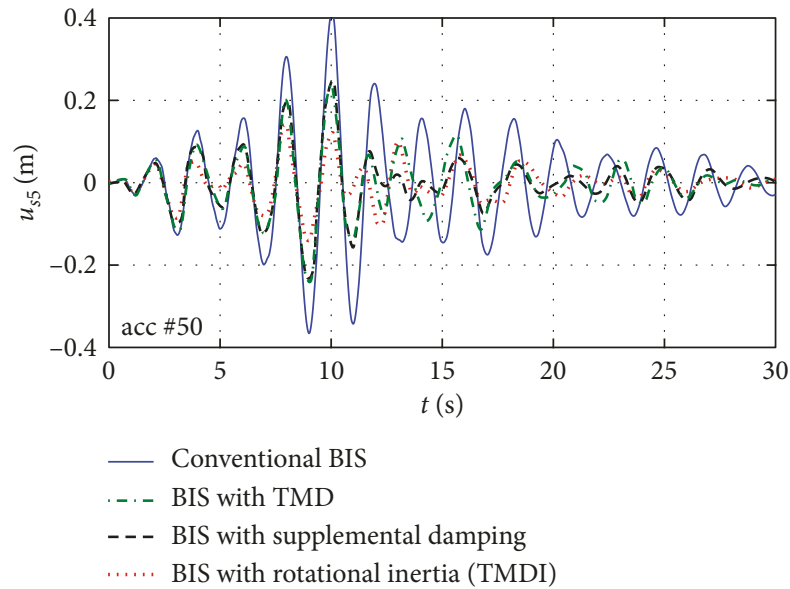

(a)

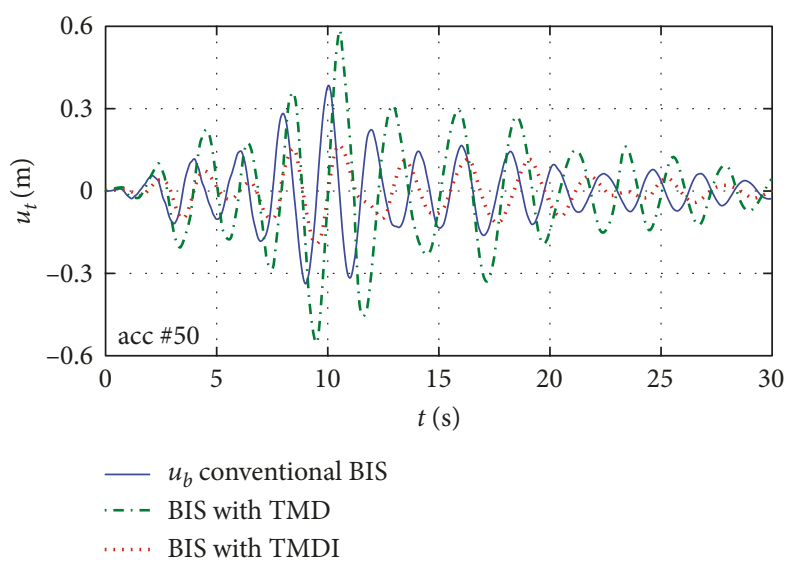

(c)

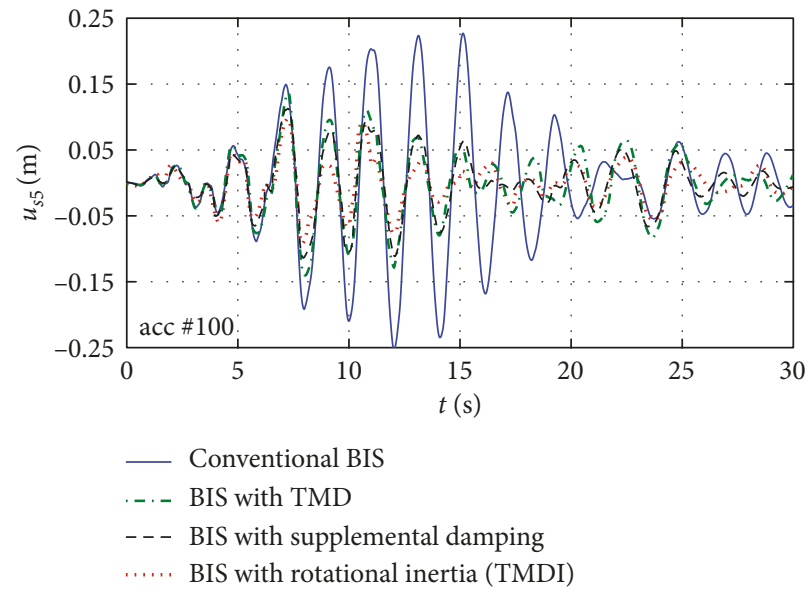

(b)

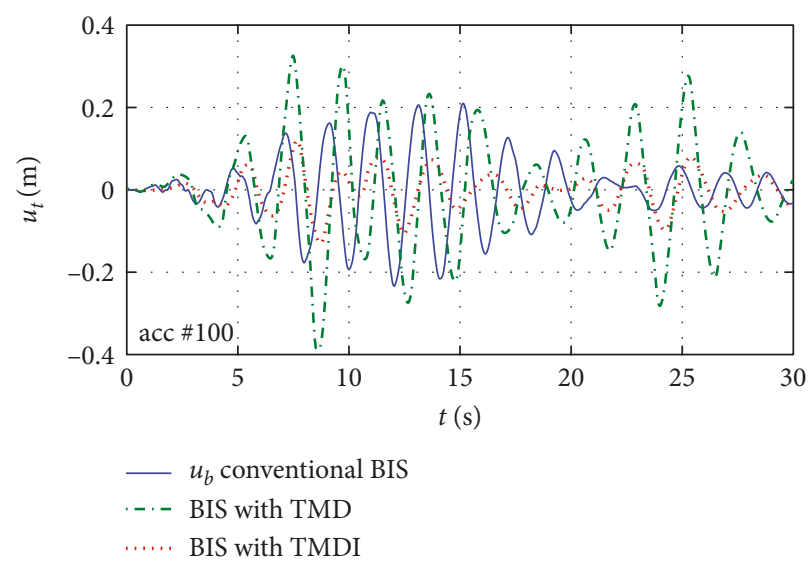

(d)

Figure 15: Time-history response of the RC building shown in Figure 14 subject to two arbitrary artificial accelerograms: (a, b) last-floor displacement; (c, d) secondary system (BIS, TMDI, TMD) displacement.

gradually so that there is time for transfer of energy from the base-isolated structure to the secondary system, which was also observed in [18]. This implies that the effectiveness of the TMDI can be jeopardized if the accelerogram has a high impulsive content in the first instants of motion [15]. Also reported in Figures 15(c) and 15(d) are the displacements of the secondary mass (TMD, TMDI). It is seen that the TMD displacements may be disproportionately large, exceeding the maximum displacement of the BIS in the uncontrolled case. On the contrary, the displacements of the TMDI are reasonably limited.

One possible drawback of the proposed system with rotational inertia could be the generated force, which is proportional to the relative acceleration. To assess the incidence of this force, in Figure 16 for the same two accelerograms discussed above, we display the time histories of the reactive forces generated in the system. In particular, we compare the damping force in the conventional isolators, computed as $F=c_{b} \dot{u}_{b}$, with the reactive force in the inerterbased device, computed as $F=b_{t} \ddot{u}_{t}$. It is found that the latter force is higher than the damping force of a low-damping system of isolators with $\zeta_{b}=0.1$ but is roughly comparable to the damping force exerted in the system \#5, that is, the BIS with supplemental damping, which however yields a poorer vibration reduction according to Tables 3 and 4. Considering this fact, it is more convenient to adopt a seismic retrofitting strategy with BIS coupled with rotational inertia rather than supplemental damping. However, it is important that all the elements that connect the inerter device to the structure must resist the forces arising from the above calculation, and therefore, the use of stiff supports is highly recommended.

The verification procedure of the constraints listed in (12) is carried out in a graphical way in Figure 17. In particular, the average maximum distribution profiles of the horizontal displacements, the inerstory drift index, and the floor accelerations are shown along the height of the building and compared to the threshold values indicated in (12), which are reported as a thicker solid line. As expected, while the displacement constraint is met by the fixed-base building, the conditions on the interstory drift and floor acceleration are largely exceeded. This means that an earthquake protection system should be adopted. The conventional BIS (with low-damping $\zeta_{b}=0.1$ ) violates the conditions on displacements and floor accelerations. 
TABLE 3: List of results considering average RMS values of response indicators (Monte Carlo method with 100 artificial samples).

\begin{tabular}{|c|c|c|c|c|c|c|c|c|}
\hline \multirow[b]{2}{*}{$\begin{array}{l}\text { Seismic retrofitting } \\
\text { strategy }\end{array}$} & \multicolumn{6}{|c|}{ Superstructure-related response indicators } & \multirow[b]{2}{*}{$\begin{array}{c}\text { BIS } \\
\text { displacement } \\
u_{b}(\mathrm{~m}) \cdot 10^{-2}\end{array}$} & \multirow[b]{2}{*}{$\begin{array}{c}\text { TMD } \\
\text { stroke } \\
u_{t}(\mathrm{~m}) \cdot 10^{-2}\end{array}$} \\
\hline & $\begin{array}{c}\text { Last-floor } \\
\text { displacement } \\
u_{s 5}(\mathrm{~m}) \cdot 10^{-2}\end{array}$ & $\begin{array}{l}\text { 2nd interstory } \\
\text { drift } \\
\Delta u_{s 2}(\mathrm{~m}) \cdot 10^{-4} \\
\end{array}$ & $\begin{array}{c}\text { 4th floor } \\
\text { acceleration } \\
\ddot{u}_{s 4}\left(\mathrm{~m} / \mathrm{s}^{2}\right) \cdot 10^{-2}\end{array}$ & $\begin{array}{c}\text { Base } \\
\text { shear } \\
V_{b}(\mathrm{kN})\end{array}$ & $\begin{array}{c}\text { Kinetic } \\
\text { energy } \\
T_{s}(\mathrm{kNm})\end{array}$ & $\begin{array}{c}\text { Strain } \\
\text { energy } \\
E_{s}(\mathrm{Nm})\end{array}$ & & \\
\hline $\begin{array}{l}\text { \#1. Conventional } \\
\text { BIS } \\
\text { (reference solution) }\end{array}$ & 8.58 & 17.27 & 82.31 & 229.0 & 38.19 & 1879.8 & 7.92 & - \\
\hline $\begin{array}{l}\# 2 . \text { BIS with } \\
\text { rotational } \\
\text { inertia (TMDI) }\end{array}$ & $\begin{array}{c}3.87 \\
(-54.8 \%)\end{array}$ & $\begin{array}{c}8.20 \\
(-52.5 \%)\end{array}$ & $\begin{array}{c}42.34 \\
(-48.6 \%)\end{array}$ & $\begin{array}{c}108.0 \\
(-53.1 \%)\end{array}$ & $\begin{array}{c}9.88 \\
(-74.1 \%)\end{array}$ & $\begin{array}{c}502.5 \\
(-73.3 \%)\end{array}$ & $\begin{array}{c}3.56 \\
(-55.1 \%)\end{array}$ & $\begin{array}{c}5.85 \\
(-26.1 \%)\end{array}$ \\
\hline \#3. BIS with TMD & $\begin{array}{c}5.61 \\
(-34.7 \%)\end{array}$ & $\begin{array}{c}11.02 \\
(-36.2 \%)\end{array}$ & $\begin{array}{c}53.95 \\
(-34.5 \%)\end{array}$ & $\begin{array}{c}146.0 \\
(-36.4 \%)\end{array}$ & $\begin{array}{c}18.18 \\
(-52.4 \%)\end{array}$ & $\begin{array}{c}844.9 \\
(-55.1 \%)\end{array}$ & $\begin{array}{c}5.19 \\
(-34.6 \%)\end{array}$ & $\begin{array}{c}16.41 \\
(+\mathbf{1 0 7 . 1 \%})\end{array}$ \\
\hline $\begin{array}{l}\text { \#4. BIS with } \\
\text { supplemental } \\
\text { damping }\end{array}$ & $\begin{array}{c}5.06 \\
(-41.0 \%)\end{array}$ & $\begin{array}{c}10.48 \\
(-39.3 \%)\end{array}$ & $\begin{array}{c}52.17 \\
(-36.6 \%)\end{array}$ & $\begin{array}{c}138.0 \\
(-39.6 \%)\end{array}$ & $\begin{array}{c}15.73 \\
(-58.8 \%)\end{array}$ & $\begin{array}{c}772.6 \\
(-58.9 \%)\end{array}$ & $\begin{array}{c}4.67 \\
(-41.2 \%)\end{array}$ & - \\
\hline $\begin{array}{l}\text { \#5. BIS with } \\
\text { supplemental } \\
\text { damping }\end{array}$ & $\begin{array}{c}4.40 \\
(-48.7 \%)\end{array}$ & $\begin{array}{c}10.48 \\
(-39.3 \%)\end{array}$ & $\begin{array}{c}52.17 \\
(-36.6 \%)\end{array}$ & $\begin{array}{c}122.0 \\
(-47.0 \%)\end{array}$ & $\begin{array}{c}15.73 \\
(-58.8 \%)\end{array}$ & $\begin{array}{c}772.6 \\
(-58.9 \%)\end{array}$ & $\begin{array}{c}4.00 \\
(-49.5 \%)\end{array}$ & - \\
\hline
\end{tabular}

TABLE 4: List of results considering average MAX values of response indicators (Monte Carlo method with 100 artificial samples).

\begin{tabular}{|c|c|c|c|c|c|c|c|c|}
\hline \multirow[b]{2}{*}{$\begin{array}{l}\text { Seismic retrofitting } \\
\text { strategy }\end{array}$} & \multicolumn{6}{|c|}{ Superstructure-related response indicators } & \multirow[b]{2}{*}{$\begin{array}{c}\text { BIS } \\
\text { displacement } \\
u_{b}(\mathrm{~m}) \cdot 10^{-2}\end{array}$} & \multirow[b]{2}{*}{$\begin{array}{c}\text { TMD } \\
\text { stroke } \\
u_{t}(\mathrm{~m}) \cdot 10^{-2}\end{array}$} \\
\hline & $\begin{array}{c}\text { Last-floor } \\
\text { displacement } \\
u_{s 5}(\mathrm{~m}) \cdot 10^{-2}\end{array}$ & $\begin{array}{c}2 \text { nd interstory } \\
\text { drift } \\
\Delta u_{s 2}(\mathrm{~m}) \cdot 10^{-4}\end{array}$ & $\begin{array}{c}\text { 4th floor } \\
\text { acceleration } \\
\ddot{u}_{s 4}\left(\mathrm{~m} / \mathrm{s}^{2}\right) \cdot 10^{-2}\end{array}$ & $\begin{array}{c}\text { Base } \\
\text { shear } \\
V_{b}(\mathrm{kN})\end{array}$ & $\begin{array}{c}\text { Kinetic } \\
\text { energy } \\
T_{s}(\mathrm{kNm})\end{array}$ & $\begin{array}{c}\text { Strain } \\
\text { energy } \\
E_{s}(\mathrm{Nm})\end{array}$ & & \\
\hline $\begin{array}{l}\text { \#1. Conventional BIS } \\
\text { (reference solution) }\end{array}$ & 23.67 & 48.23 & 258.32 & 634.0 & 182.05 & 8195.1 & 21.81 & - \\
\hline$\# 2$. BIS with & 13.59 & 30.37 & 183.59 & 390.0 & 70.31 & 3201.7 & 12.39 & 18.47 \\
\hline $\begin{array}{l}\text { rotational } \\
\text { inertia (TMDI) }\end{array}$ & $(-42.6 \%)$ & $(-37.0 \%)$ & $(-28.9 \%)$ & $(-38.6 \%)$ & $(-61.3 \%)$ & $(-60.9 \%)$ & $(-43.2 \%)$ & $(-15.3 \%)$ \\
\hline \#3. BIS with TMD & $\begin{array}{c}18.25 \\
(-22.9 \%)\end{array}$ & $\begin{array}{c}36.16 \\
(-25.0 \%)\end{array}$ & $\begin{array}{c}208.17 \\
(-19.4 \%)\end{array}$ & $\begin{array}{c}474.0 \\
(-25.3 \%)\end{array}$ & $\begin{array}{c}113.5 \\
(-37.7 \%)\end{array}$ & $\begin{array}{c}4512.6 \\
(-44.9 \%)\end{array}$ & $\begin{array}{c}16.85 \\
(-22.8 \%)\end{array}$ & $\begin{array}{c}48.16 \\
(+\mathbf{1 2 0 . 8})\end{array}$ \\
\hline \#4. BIS with & 16.32 & 34.89 & 204.5 & 452.3 & 97.65 & 4208.1 & 14.96 & - \\
\hline $\begin{array}{l}\text { supplemental } \\
\text { damping }\end{array}$ & $(-31.0 \%)$ & $(-27.6 \%)$ & $(-20.8 \%)$ & $(-28.8 \%)$ & $(-46.2 \%)$ & $(-48.6 \%)$ & $(-31.4 \%)$ & - \\
\hline \#5. BIS with & 14.65 & 34.89 & 204.5 & 416.0 & 97.65 & 4208.1 & 13.29 & - \\
\hline $\begin{array}{l}\text { supplemental } \\
\text { damping }\end{array}$ & $(-38.1 \%)$ & $(-27.6 \%)$ & $(-20.8 \%)$ & $(-34.4 \%)$ & $(-46.2 \%)$ & $(-48.6 \%)$ & $(-39.1 \%)$ & - \\
\hline
\end{tabular}

Increasing the BIS damping up to $\zeta_{b}=0.4$, thus considering a BIS with supplemental damping, reduces the displacements down to an acceptable level, lower than the threshold value, but the floor accelerations are still larger than the maximum admissible ones. On the contrary, the BIS with rotational inertia satisfies all the three constraints.

The analysis discussed above has been carried out by means of uniformly modulated accelerograms, as an idealization of real accelerograms that are actually fully nonstationary in nature. To corroborate the conclusions of the present investigation, we repeat the analysis with two natural earthquake ground motions to scrutinize the effectiveness of the seismic retrofitting strategies while accounting for the fully nonstationary character of the seismic input. Two recorded accelerograms are selected from the Center for Engineering Strong Motion Data (http://strongmotioncenter.org/), namely, El Centro (18 May 1940, epicenter distance from recording station $17 \mathrm{~km}$ ) and Loma Prieta (17 October 1989, epicenter distance from recording station $7 \mathrm{~km}$ ) ground motions. Relevant results are presented in Figure 18 for the time histories of the last-floor displacement response and in Figure 19 in terms of distribution profiles of displacements, interstory drift index, and floor accelerations. Despite the different seismic inputs, similar conclusions of the previous case may be drawn: once again the BIS with rotational inertia is the best seismic retrofitting strategy and outperforms the BIS with supplemental damping in the reduction of displacements, interstory drifts, and floor accelerations. A conventional, low-damping BIS is unacceptable if the conditions specified in (12) should be met, for instance, the displacements at the fifth floor exceed $20 \mathrm{~cm}$ for both the earthquakes. 


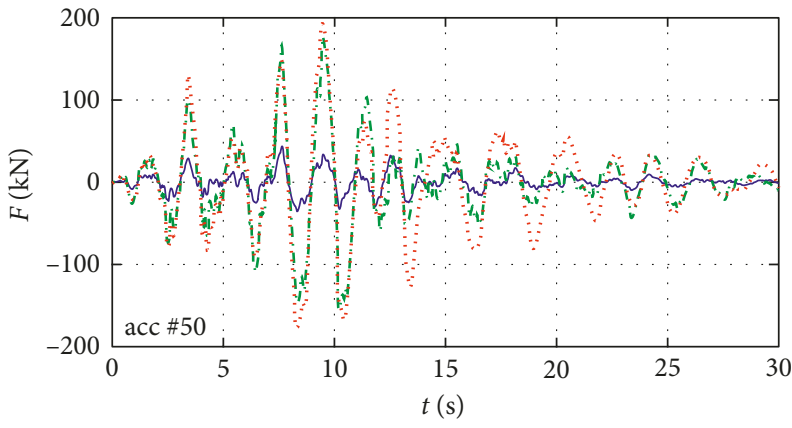

— Damping force in convertional BIS

. - . Damping force in BIS with supplemental damping

..... Inerter force in BIS with rotational inertia

(a)

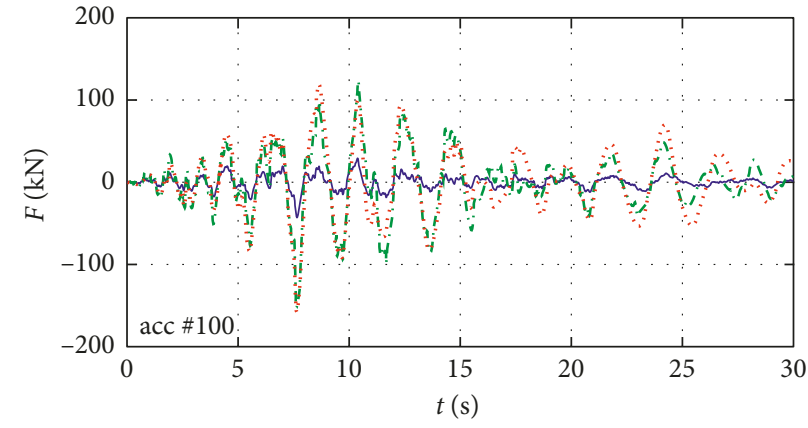

— Damping force in convertional BIS

-. - Damping force in BIS with supplemental damping

...... Inerter force in BIS with rotational inertia

(b)

FIgURE 16: Time-history for reactive forces relevant to two arbitrary artificial accelerograms.
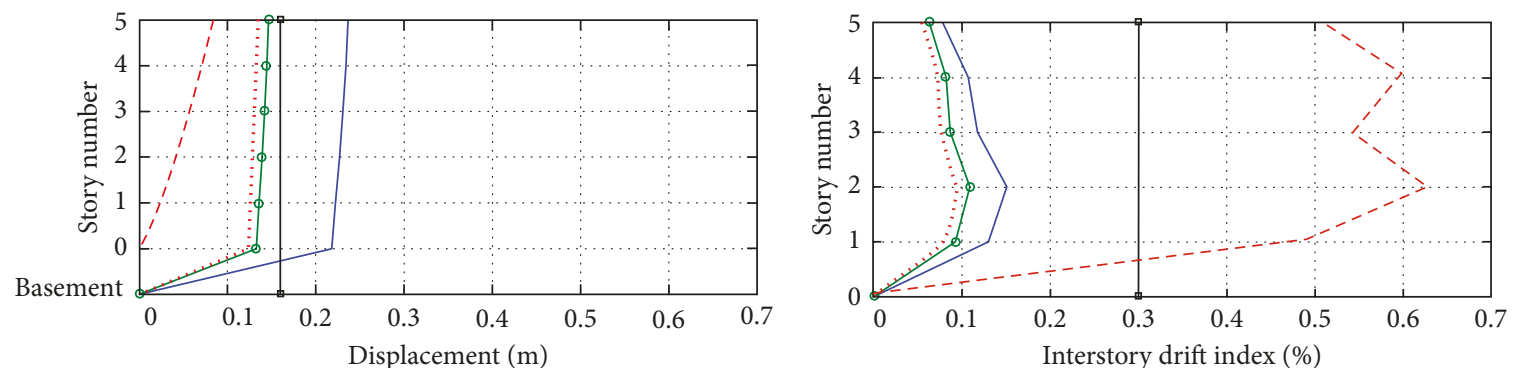

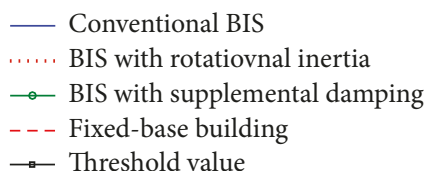

(a)
- Conventional BIS

..... BIS with rotatinal inertia

$\rightarrow$ BIS with supplemental damping

- - - Fixed-base building

$\rightarrow$ Threshold value

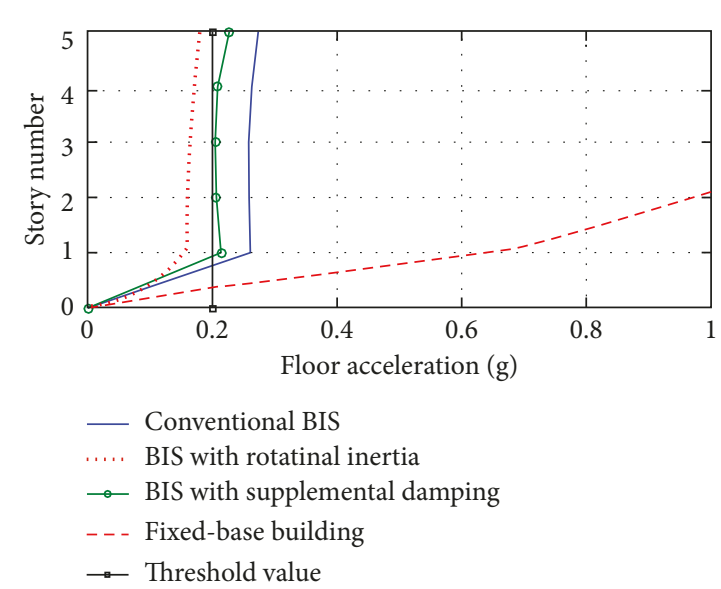

(b)

(c)

Figure 17: Monte Carlo method with 100 artificial samples: average maximum distribution of horizontal displacements (a), interstory drift index (b), and floor acceleration (c) compared to threshold values as per (12).

7.2. Impact of Tuning Failures due to Uncertainty in the Structural Parameters. The observation of real-world examples of BI solutions (see, e.g., [43, 45] and the notable case of the Christchurch Womens Hospital (CWH) in Canterbury, New Zeland [46-48], has shown that in reality base-isolated structures may not behave as expected during the occurrence 


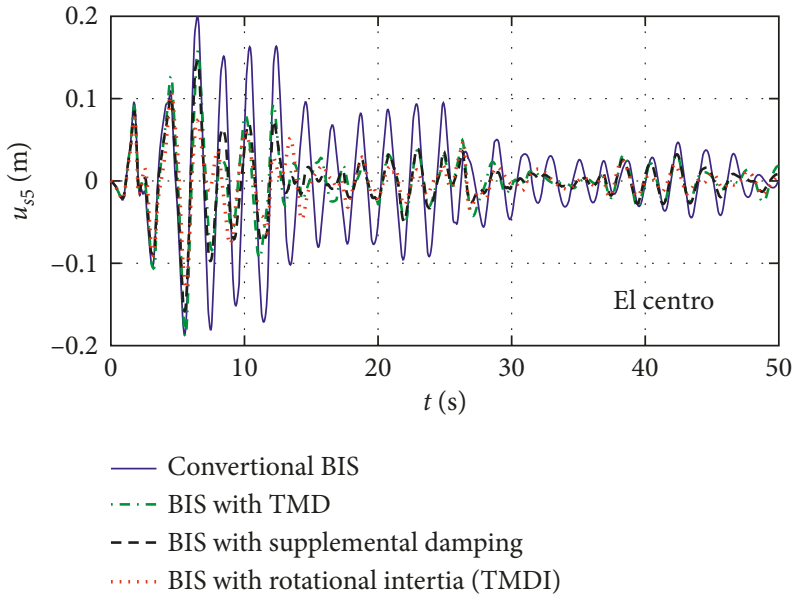

(a)

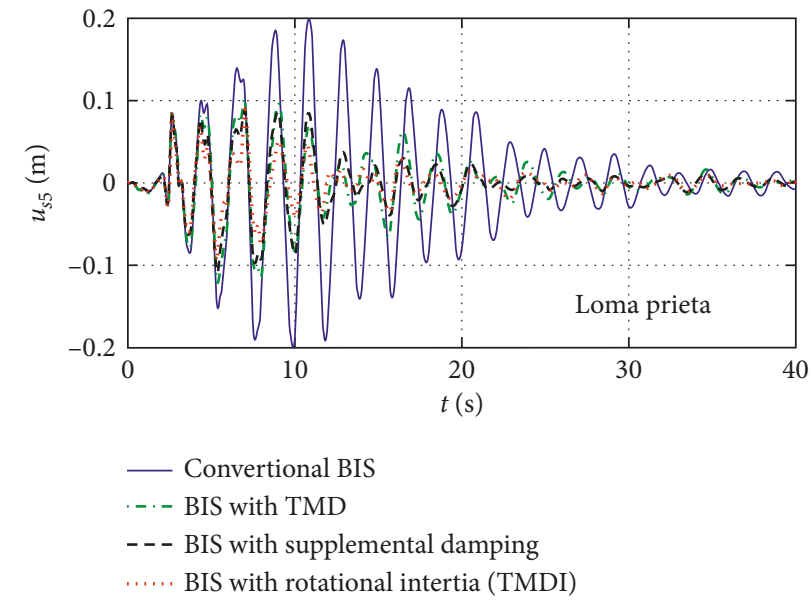

(b)

Figure 18: Last-floor displacement time-history response of the RC building shown in Figure 14 subject to El Centro (a) and Loma Prieta (b) earthquake ground motion.

of seismic events. This may be ascribed to the construction variations along with the imperfect knowledge of the structural parameters, for instance, the involved masses (due to the live loads) and the stiffness of the isolators. The as-designed and as-implemented scenarios may be quite different. As an example, in the aforementioned CWH during the Mw5.8 and Mw6.0 seismic events on December 23, 2011 [46], the yield force of the LRB isolators was higher than expected; therefore, the isolation was quite ineffective and the superstructure performed as a fixed-base building to a large extent. Actually, the isolator stiffness measured for the Mw5.8 event was approximately four times higher than the design stiffness, and very little damping was recorded unlike the expectations of the original design.

Based on these observations, the assessment of the effectiveness of the proposed BIS + TMDI solution should be made by incorporating the effect of detuning, that is, by slightly varying the dynamic properties of natural frequency (thus accounting for the variation in both masses and stiffness) and damping ratio of the TMD tuned isolators. It seems of valuable importance to scrutinize and quantify the impact of tuning failure (effects of detuning) on the achieved isolation performance.

No practical implementation of the BIS + TMDI strategy in real structures is available so far; therefore, the investigation is here carried out numerically by means of a parametric analysis. In particular, the same five-story RC building shown in Figure 14 is analyzed with the BIS + rotational inertia (TMDI system). The detected tuning parameters of the TMD isolators $\omega_{t}$ and $\zeta_{t}$ are slightly varied from their optimal values reported in Table 2. In particular, considering the previous example of the CWH [46] where the actual stiffness of the isolators was four times higher than the one assumed in the calculation, we explore a range of parameters up the extreme situation in which the natural frequency $\omega_{t}=\sqrt{k_{t} / m_{\text {eff }}}=2 \omega_{t \text { opt }}$, which corresponds to a factor four in the isolator stiffness $k_{t}$. Moreover, we also explore a similar range of variation for the damping ratio $\zeta_{t}$.
This is a simplified way to simulate what happened in the $\mathrm{CWH}$ [46] due to the discrepancies in the isolator stiffness. Furthermore, this is a simplified way to take into account the variability of the isolators' mechanical properties during the seismic event, for example, the friction coefficient of the friction pendulum isolators [49]. Relevant results are depicted in Figures 20 and 21. It can be seen that, as a confirmation of the numerical procedure proposed, the optimal tuning parameters lead to the minimization of the last-floor and the BIS displacements (cf. Figure 20). The base shear and the kinetic energy are also reported in the plots and seem to be minimized by a slightly higher value of frequency and by a higher damping ratio than the one assumed for the minimization of the displacements. An important aspect emerges from this analysis: as the tuning parameters depart from their optimal values, the structure displacements increase and the effectiveness of the isolation system decreases accordingly, which is quite reasonable. In particular, for a TMD frequency two times higher than the optimal one, the superstructure displacements increase of about $80 \%$ and thus the isolation efficacy reduces. These results are in line with the conclusions drawn for the CWH [46] and demonstrate that the isolator stiffness is a key parameter in order for the isolation to be fully effective. A wrong estimation of the TMD frequency may hamper the effectiveness of this technology over a broad bandwidth of inputs. In this regard, the solution with supplemental damping does not suffer from this drawback. Although the analyzed scenario (isolator stiffness four times higher than expected and natural frequency two times higher than expected) is quite extreme, it should be noted that purely providing damping is not affected by such issues related to detuning effects.

7.3. Critical Discussion and Alternative Systems. A variety of seismic retrofitting strategies exist, which are obviously not 

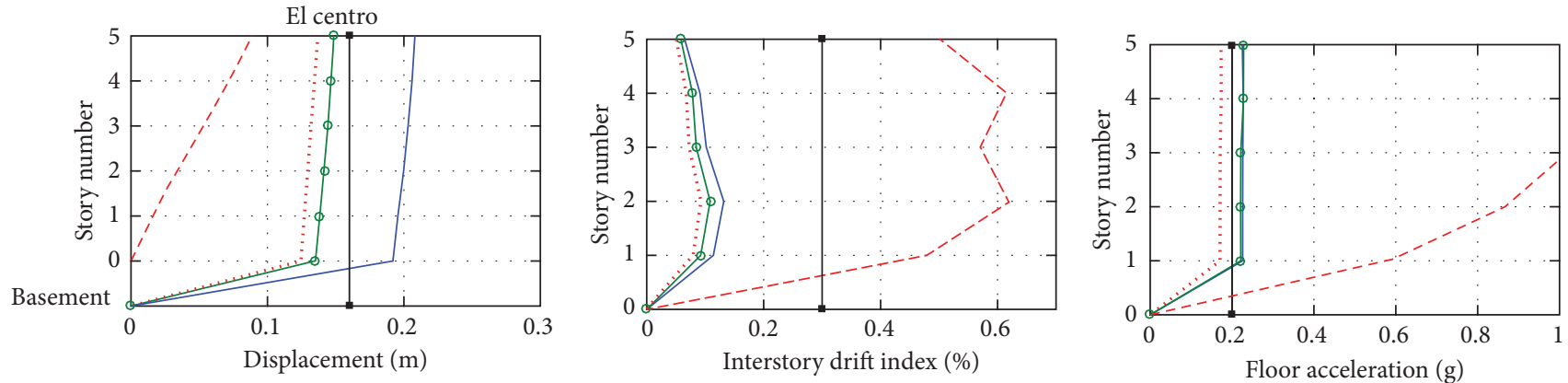

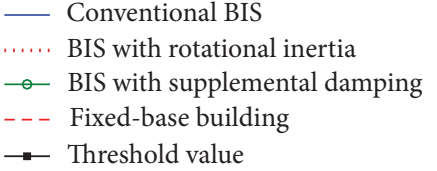

(a)

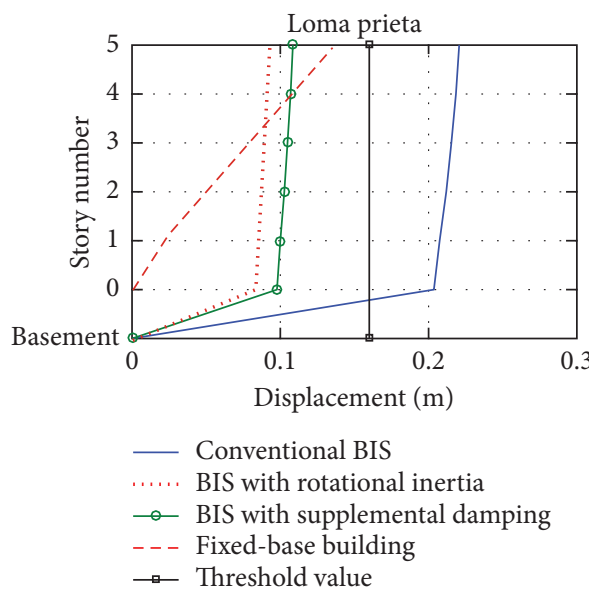

(d)

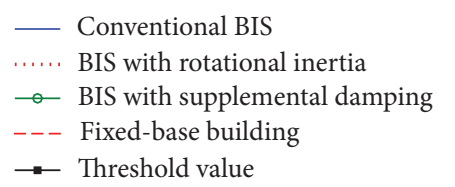

(b)

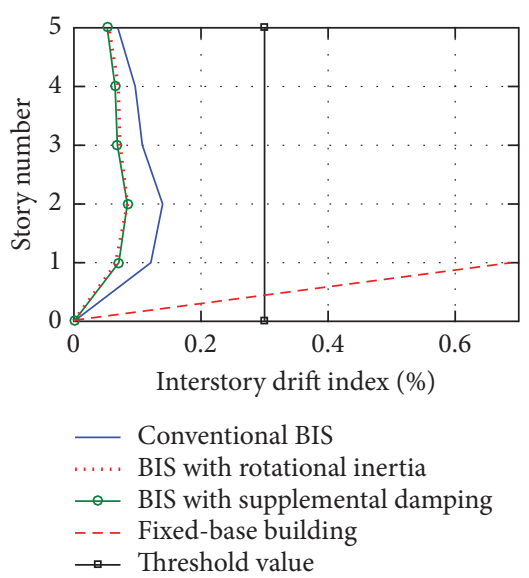

(e)

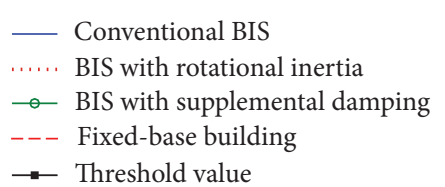

(c)

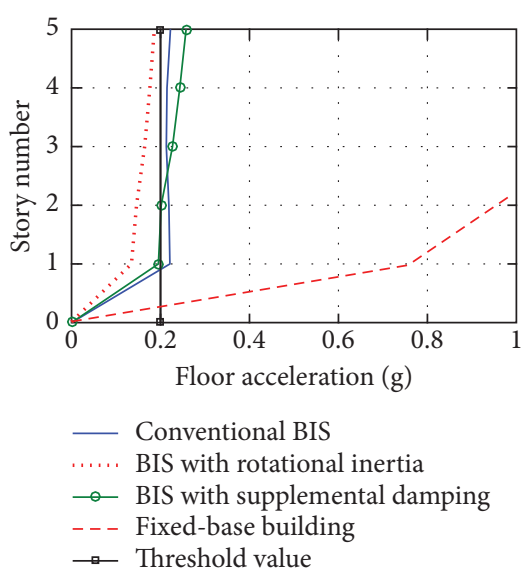

(f)

Figure 19: Average maximum distribution of horizontal displacements (a, d), interstory drift index (b, e), and floor acceleration (c, f) for El Centro and Loma Prieta earthquake ground motions.

limited to the few ones discussed in this paper. Among these strategies, we mention the coupling of adjacent buildings by means of passive damping devices to reduce the risk of pounding [50-52] and to improve the seismic performance of the two systems [53-55], the introduction of viscous dampers along the height of the building that produce desired levels of interstory drifts while reducing seismic forces $[56,57]$, the interstory isolation by the use of seismic isolators at levels other than the base along the height of a building $[58,59]$, and the interstory isolation implemented by converting masses already present on the structure into tuned masses according to a nonconventional TMD scheme $[38,60]$. Moreover, there are a range of supplemental dampers that do not have a viscous nature, for example, buckling restrained braces, yielding fuse systems, and the HF2V device based on the lead extrusion technology [61-64], to quote just a few. In these cases, the simplified assumption of a linear viscous damping, made throughout this paper, does not apply, and one should consider a more complicated constitutive behavior, which is beyond the scope of the present study. Additionally, the class of so- called semi-active devices, having variable effective stiffness and/or variable effective damping, provides supplemental damping while keeping the overall base shear limited. In this context, resettable stiffness devices using electrorheological and magnetorheological materials have been increasingly developed [65-67] and studied in large-scale experimental validations in an attempt to optimize damping and minimize response by effectively reshaping and customizing the hysteretic loops (see, e.g., [68-71]). The nonlinearity of these models, including valve size, mass flow rate, and friction, has been investigated in experiments and numerical models [72]. Other types of nonlinear viscous dampers are the directionand displacement-dependent (D3) devices proposed in [73] that are entirely passive, thus representing more robust and lower cost devices. These are just a few devices and technologies and the list is far from being exhaustive (see, e.g., $[74,75]$ for an overview). This very long list underlines that the present analysis has only focused on a quite limited set of intervention strategies, making some simplifying assumptions. Further investigation is certainly needed to address the problem in a more comprehensive fashion, 


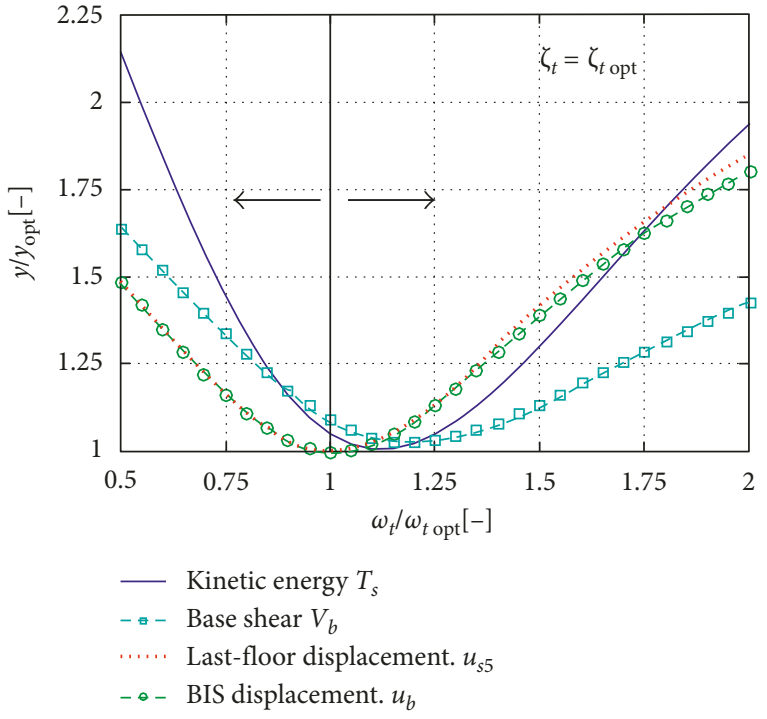

(a)

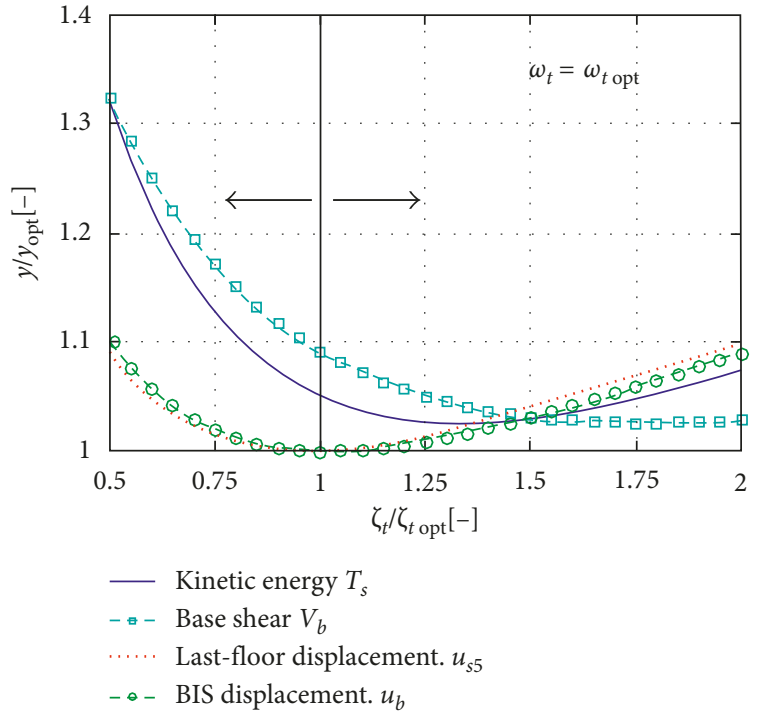

(b)

FIGURE 20: Impact of tuning failure due to imperfect knowledge of the structural TMD dynamic parameters: effect on four response indicators.

by extending and generalizing the results here found. Nevertheless, a few advantages of the proposed technique combining seismic base isolation with supplemental rotational inertia over alternative strategies, at least those dealt with in the present analysis, may be recognized as follows:

(1) The retrofitting operations are limited just to the ground floor level or to the basement level, thus not interfering with the architectural aspects of the building along its height, and the remaining parts of the structure do not have to be modified and/or altered (unlike other systems applied to the structural elements directly (see, e.g., [76, 77])).

(2) It is well known that the seismic base isolation permits a strong reduction of forces to the superstructure so that the building can be designed to remain in the elastic range. Consequently, limited damage is expected in the superstructure, thus avoiding interruption of operational and functional aspects of the building, while plasticnonlinear behavior is concentrated at the isolation level. This consideration, stated for the conventional BIS, also applies to the improved BIS with supplemental rotational inertia. In other words, during and after the earthquake, the structure can, in principle, continue to hold its function, which is of extreme importance for strategic buildings having civil and social duties.

(3) As compared to the conventional base isolation scheme, the displacements are drastically reduced in the proposed BIS with supplemental rotational inertia, which is essential in existing structures with limited seismic joints. Moreover, it has been found that also interstory drifts (related to the internal stress of the frame elements) and floor accelerations are reduced by the proposed strategy, much more than employing supplemental damping.

(4) The inerter is a quite compact mechanical device that can be designed to provide any desired value of inertance. On the contrary, effective vibration reduction is achieved by the TMD provided that large amounts of mass are introduced into the system. Unless an architectural function is assigned to such an additional mass (e.g., a parking space and utilities room), the feasibility and cost-effectiveness of this retrofitting strategy may be hampered in practical applications because of economic reasons.

(5) The use of the TMD as a retrofitting strategy is also hindered by the TMD strokes, which may be unacceptably large, thus requiring a large space/clearance in the building in order to accommodate the displacement demand of this secondary mass/subsystem, sometimes even exceeding the maximum admissible displacement of the BIS.

(6) As compared to alternative retrofitting strategies comprising fluid viscous dampers installed along the height of the building, the proposed system can accommodate large relative displacements without suffering from the issues of viscous heating and potential leaking that challenge the implementation of fluid dampers [16].

Nevertheless, all the encouraging outcomes arisen from this study should be better verified before a practical implementation of this system for seismic retrofitting purposes can be accomplished. For instance, while the assumption of linear viscous damping for the superstructure is reasonable, it would be advisable to extend this research 


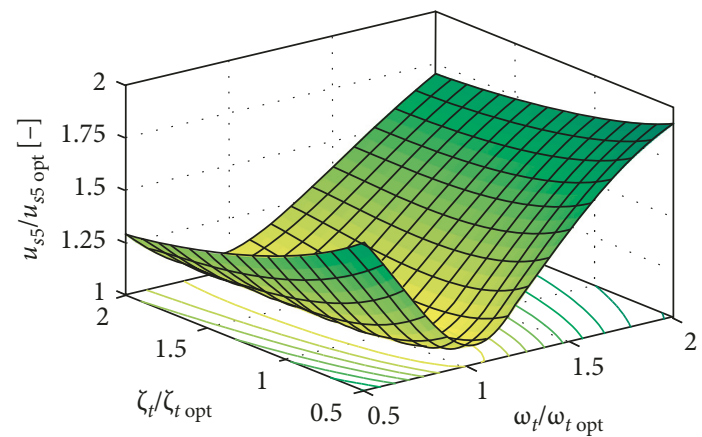

(a)

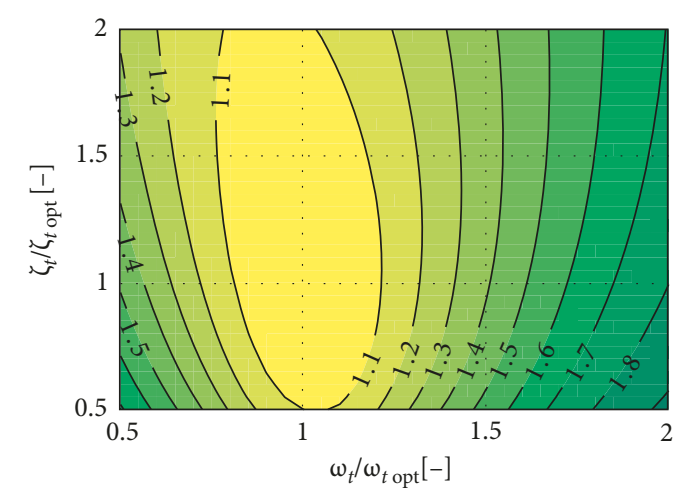

(c)

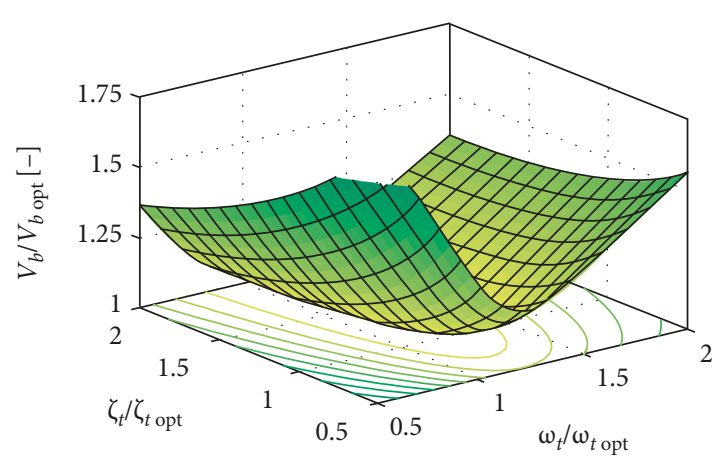

(b)

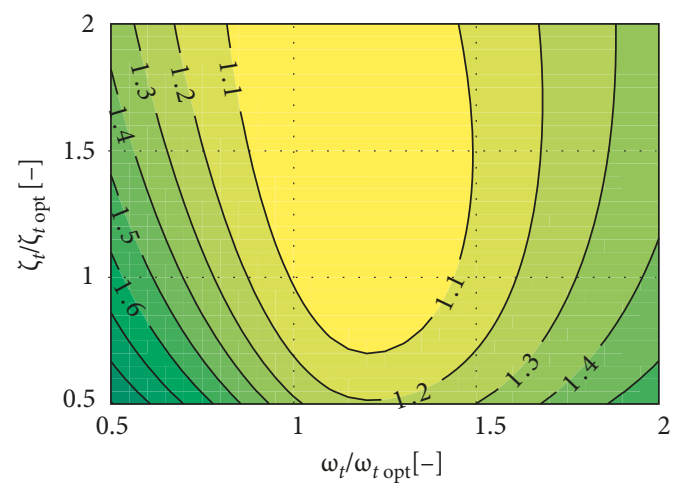

(d)

FIGURE 21: Impact of tuning failure due to imperfect knowledge of the structural TMD dynamic parameters: surface and contour plots of the last-floor displacement $(a, c)$ and of the base shear $(b, d)$, respectively.

work to incorporate a more appropriate, realistic nonlinear behavior of the isolators, thus accounting for their actual inelastic characteristics. However, it has been demonstrated that the system performs at its best when low-damping (rubber bearing) isolators are considered; therefore, the equivalent linear viscous damping idealization dealt with in this paper could represent a reasonable assumption at least for preliminary design purposes. On the other hand, the auxiliary isolators are medium-to-high damping isolators, which implies that a better description of their nonlinear behavior is recommended. This task is left for future research work.

\section{Concluding Remarks}

Existing structures having a limited seismic joint and requiring a high level of earthquake protection, such as building with strategic importance, have been addressed in this paper. To limit the forces in the structure and to keep the functional and operational aspects of these buildings during and after earthquakes, the strategy of seismic base isolation has been assumed as the starting point of a seismic retrofitting process. On the other hand, the limited seismic joint of several existing structures built in the past requires careful considerations on the displacement demand of the baseisolated structure. In this paper, two alternative remedies are discussed and compared, the former combining a base isolation system with supplemental damping and the latter coupling the base isolation with supplemental rotational inertia via the use of the TMDI, which is a TMD with inerter. The combination of the base isolation system with a TMD has retrieved a special case of the system with the TMDI for a zero value of the inertance. Optimal design of the system and the influence of the soil characteristics on the best tuning parameters have been dealt with. A practical strategy of implementation of the proposed system into existing structures is presented, which comprises the use of two sets of isolators, namely, some conventional isolators with lowdamping properties and some auxiliary isolators that are more flexible and equipped with medium-to-high damping characteristics. The inerter is connected in between the auxiliary isolators and the ground. Time-history analyses for a simple multistory building subject to both artificial accelerograms and natural earthquake ground motions have been performed. The basic steps involved in a seismic retrofitting procedure via the proposed technique are outlined. A variety of response indicators are computed that are useful and of practical importance for design engineers, including the base shear, interstory drifts, floor accelerations, kinetic energy, and so forth. It is found that the BIS with supplemental rotational inertia is a more effective seismic retrofitting strategy than the BIS with supplemental damping for all the response indicators above mentioned, at least for the cases here analyzed. Advantages of the proposed techniques over a few other approaches in the literature have also been critically discussed but only from a qualitative point of view. 
Sensitivity analysis and the impact of tuning failures due to imperfect knowledge of the structural parameters (e.g., the TMD mass and the stiffness of the isolators) have also been discussed, motivated by the observation of a real-world case example of isolation [46].

Despite many simplifying assumptions adopted in this paper and the simplicity of the structural model analyzed, the authors' opinion is that the BIS with supplemental rotational inertia can be considered as a feasible, low-mass, and efficient seismic retrofitting strategy of existing structures with limited seismic joint. However, before a practical implementation can be done, prototype testing and/or experimental findings are essential to gain a more accurate understanding of this system and to to validate the idealized models commonly adopted in the literature.

\section{Conflicts of Interest}

The authors declare that they have no conflicts of interest.

\section{Acknowledgments}

The financial support from the Italian Ministry of Education, University and Research (PRIN Grant 2015TTJN95- "Identification and monitoring of complex structural systems"), is gratefully acknowledged. The first author wishes to express his personal gratitude to this research fund and also to the PRIN Grant 2015JW9NJT, through which a postdoctoral scholarship was awarded.

\section{References}

[1] F. Naeim and J. M. Kelly, Design of Seismic Isolated Structures: From Theory to Practice, John Wiley \& Sons, New York, NY, USA, 1999.

[2] V. Zayas, S. Low, and S. Mahin, "The FPS earthquake resisting system," Report No. CB/EERC-87/01, Earthquake Engineering Research Center, University of California, Berkeley, CA, USA, 1987.

[3] M. D. Symans, F. A. Charney, A. S. Whittaker et al., "Energy dissipation systems for seismic applications: current practice and recent developments," Journal of Structural Engineering, vol. 134, no. 1, pp. 3-21, 2008.

[4] T. T. Soong and G. F. Dargush, Passive Energy Dissipation Systems in Structural Engineering, John Wiley \& Sons, Chichester, UK, 1997.

[5] S. A. Anagnostopoulos and K. V. Spiliopoulos, "An investigation of earthquake induced pounding between adjacent buildings," Earthquake Engineering \& Structural Dynamics, vol. 21, no. 4, pp. 289-302, 1992.

[6] T. H. Heaton, J. F. Hall, D. J. Wald, and M. W. Halling, "Response of high-rise and base-isolated buildings to a hypothetical Mw 7.0 blind thrust earthquake," Science, vol. 267, no. 5195, pp. 206-211, 1995.

[7] T. Ariga, Y. Kanno, and I. Takewaki, "Resonant behaviour of base-isolated high-rise buildings under long-period ground motions," Structural Design of Tall and Special Buildings, vol. 15, no. 3, pp. 325-338, 2006.

[8] I. Takewaki, S. Murakami, K. Fujita, S. Yoshitomi, and M. Tsuji, "The 2011 off the Pacific coast of Tohoku earthquake and response of high-rise buildings under long-period ground motions," Soil Dynamics and Earthquake Engineering, vol. 31, no. 11, pp. 1511-1528, 2011.

[9] J. M. Kelly, "The role of damping in seismic isolation," Earthquake Engineering \& Structural Dynamics, vol. 28, no. 1, pp. 3-20, 1999.

[10] J. P. Den Hartog, Mechanical Vibrations, McGraw-Hill, New York, NY, USA, 1934.

[11] M. C. Smith, "Synthesis of mechanical networks: the inerter," IEEE Transactions on Automatic Control, vol. 47, no. 10, pp. 1648-1662, 2002.

[12] M. Z. Q. Chen, C. Papageorgiou, F. Scheibe, F.-C. Wang, and M. C. Smith, "The missing mechanical circuit element," IEEE Circuits and Systems Magazine, vol. 9, no. 1, pp. 10-26, 2009.

[13] I. F. Lazar, S. A. Neild, and D. J. Wagg, "Using an inerter-based device for structural vibration suppression," Earthquake Engineering \& Structural Dynamics, vol. 43, no. 8, pp. 11291147, 2014.

[14] L. Marian and A. Giaralis, "Optimal design of a novel tuned mass-damper-inerter (TMDI) passive vibration control configuration for stochastically support-excited structural systems," Probabilistic Engineering Mechanics, vol. 38, pp. 156-164, 2014.

[15] D. Pietrosanti, M. De Angelis, and M. Basili, "Optimal design and performance evaluation of systems with Tuned Mass Damper Inerter (TMDI)," Earthquake Engineering \& Structural Dynamics, vol. 46, no. 8, pp. 1367-1388, 2017.

[16] N. Makris and G. Kampas, "Seismic protection of structures with supplemental rotational inertia," Journal of Engineering Mechanics, vol. 142, no. 11, p. 04016089, 2016.

[17] J. N. Yang, A. Danielians, and S. C. Liu, "A seismic hybrid control systems for building structures," Journal of Engineering Mechanics, vol. 117, no. 4, pp. 836-853, 1991.

[18] T. Taniguchi, A. Der Kiureghian, and M. Melkumyan, "Effect of tuned mass damper on displacement demand of baseisolated structures," Engineering Structures, vol. 30, no. 12, pp. 3478-3488, 2008.

[19] H. C. Tsai, "The effect of tuned-mass dampers on the seismic response of base-isolated structures," International Journal of Solids and Structures, vol. 32, no. 8-9, pp. 1195-1210, 1995.

[20] P. Xiang and A. Nishitani, "Optimum design for more effective tuned mass damper system and its application to baseisolated buildings," Structural Control and Health Monitoring, vol. 21, no. 1, pp. 98-114, 2014.

[21] K. Saito, K. Yogo, Y. Sugimura, S. Nakaminami, and K. Park, "Application of rotary inertia to displacement reduction for vibration control system," in Proceedings of 13th World Conference on Earthquake Engineering, p. 13, Vancouver, BC, Canada, 2004.

[22] M. Saitoh, "On the performance of gyro-mass devices for displacement mitigation in base isolation systems," Structural Control and Health Monitoring, vol. 19, no. 2, pp. 246-259, 2012.

[23] D. De Domenico, N. Impollonia, and G. Ricciardi, "Soildependent optimum design of a new passive vibration control system combining seismic base isolation with tuned inerter damper," Soil Dynamics and Earthquake Engineering, vol. 105, pp. 37-53, 2018.

[24] D. De Domenico and G. Ricciardi, "An enhanced base isolation system equipped with optimal Tuned Mass Damper Inerter (TMDI)," Earthquake Engineering \& Structural Dynamics, pp. 1-24, 2017.

[25] T. Hashimoto, K. Fujita, M. Tsuji, and I. Takewaki, "Innovative base-isolated building with large mass-ratio TMD at 
basement for greater earthquake resilience," Future Cities and Environment, vol. 1, no. 9, pp. 1-19, 2015.

[26] European Standard, Eurocode 8: Design of Structures for Earthquake Resistance - Part 1: General Rules, Seismic Actions and Rules for Buildings, European Committee for Standardisation, Brussels, Belgium, 2003.

[27] J. Salvi and E. Rizzi, "Optimum tuning of Tuned Mass Dampers for frame structures under earthquake excitation," Structural Control and Health Monitoring, vol. 22, no. 4, pp. 707-725, 2015.

[28] J. B. Chen, W. Liu, Y. Peng, and J. Li, "Stochastic seismic response and reliability analysis of base-isolated structures," Journal of Earthquake Engineering, vol. 11, no. 6, pp. 903-924, 2007.

[29] M. B. Priestley, "Evolutionary spectra and non-stationary processes," Journal of the Royal Statistical Society: Series B, vol. 27, no. 2, pp. 204-237, 1965.

[30] A. Giaralis and P. D. Spanos, "Derivation of response spectrum compatible non-stationary stochastic processes relying on Monte Carlo-based peak factor estimation," Earthquakes and Structures, vol. 3, no. 3-4, pp. 581-609, 2012.

[31] T. I. Hsu and M. C. Bernard, "A random process for earthquake simulation," Earthquake Engineering \& Structural Dynamics, vol. 6, no. 4, pp. 347-362, 1978.

[32] R. W. Clough and J. Penzien, Dynamics of Structures, Computers and Structures Inc., Berkeley, CA, USA, 3rd edition, 2003.

[33] P. Castaldo and M. Ripani, "Optimal design of friction pendulum system properties for isolated structures considering different soil conditions," Soil Dynamics and Earthquake Engineering, vol. 90, pp. 74-87, 2016.

[34] K. Kanai, "Semi-empirical formula for the seismic characteristics of the ground motion," Bulletin of the Earthquake Research Institute, vol. 35, no. 2, pp. 309-325, 1957.

[35] H. Tajimi, "A statistical method of determining the maximum response of a building structure during an earthquake," in Proceedings of the 2nd World Conference on Earthquake Engineering, vol. 2, pp. 781-798, Tokyo, Japan, 1960.

[36] H. Buchholdt, Structural Dynamics for Engineering, Thomas Teldfort, London, UK, 1997.

[37] A. Der Kiureghian and A. Neuenhofer, "Response spectrum method for multi-support seismic excitations," Earthquake Engineering \& Structural Dynamics, vol. 21, no. 8, pp. 713740, 1992.

[38] A. Reggio and M. De Angelis, "Optimal energy-based seismic design of non-conventional Tuned Mass Damper (TMD) implemented via inter-story isolation," Earthquake Engineering \& Structural Dynamics, vol. 44, no. 10, pp. 16231642, 2015.

[39] European Standard, Eurocode 8: Design of Structures for Earthquake Resistance - Part 3: Assessment and Retrofitting of Buildings, European Committee for Standardisation, Brussels, Belgium, 2005.

[40] D. De Domenico, "RC members strengthened with externally bonded FRP plates: a FE-based limit analysis approach," Composites Part B: Engineering, vol. 71, pp. 159-174, 2015.

[41] A. K. Chopra, Dynamics of Structures, Pearson Prentice Hall, Upper Saddle River, NJ, USA, 4th edition, 2012.

[42] M. Shinozuka and G. Deodatis, "Simulation of stochastic processes by spectral representation," Applied Mechanics Reviews, vol. 44, no. 4, pp. 191-203, 1991.

[43] T. Guo, W. Xu, L. Song, and L. Wei, "Seismic-isolation retrofits of school buildings: practice in China after recent devastating earthquakes," Journal of Performance of Constructed Facilities, vol. 28, no. 1, pp. 96-107, 2014.

[44] F. Sadek, B. Mohraz, A. W. Taylor, and R. M. Chung, "A method of estimating the parameters of Tuned Mass Dampers for seismic applications," Earthquake Engineering \& Structural Dynamics, vol. 26, no. 6, pp. 617-635, 1997.

[45] N. Lakshmanan, K. Sathish Kumar, R. Sreekala, K. Muthumani, J. Guru, and N. Gopalakrishnan, "Experimental investigations on the seismic response of a base-isolated reinforced concrete frame model," Journal of Performance of Constructed Facilities, vol. 22, no. 5, pp. 289-296, 2008.

[46] A. Kuang, A. Sridhar, J. Garven et al., "Christchurch Women's Hospital: performance analysis of the base-isolation system during the series of canterbury earthquakes 2011-2012," Journal of Performance of Constructed Facilities, vol. 30, no. 4, p. 04015096, 2015.

[47] A. Sridhar, A. Kuang, J. Garven et al., "Christchurch Women's Hospital: analysis of measured earthquake data during the 2011-2012 christchurch earthquakes," Earthquake Spectra, vol. 30, no. 1, pp. 383-400, 2014.

[48] C. Zhou, J. G. Chase, G. W. Rodgers, A. Kuang, S. Gutschmidt, and C. Xu, "Performance evaluation of CWH base isolated building during two major earthquakes in christchurch," Bulletin of the New Zealand Society for Earthquake Engineering, vol. 48, no. 4, pp. 264-273, 2015.

[49] D. De Domenico, G. Ricciardi, and G. Benzoni, "Analytical and finite element investigation on the thermo-mechanical coupled response of friction isolators under bidirectional excitation," Soil Dynamics and Earthquake Engineering, vol. 106, pp. 131-147, 2018.

[50] B. D. Westermo, "The dynamics of interstructural connection to prevent pounding," Earthquake Engineering \& Structural Dynamics, vol. 18, no. 5, pp. 687-699, 1989.

[51] M. Barbato and E. Tubaldi, "A probabilistic performancebased approach for mitigating the seismic pounding risk between adjacent buildings," Earthquake Engineering \& Structural Dynamics, vol. 42, no. 8, pp. 1203-1219, 2013.

[52] E. Tubaldi, "Dynamic behavior of adjacent buildings connected by linear viscous/viscoelastic dampers," Structural Control and Health Monitoring, vol. 22, no. 8, pp. 1086-1102, 2015.

[53] C. C. Patel and R. S. Jangid, "Dynamic response of identical adjacent structures connected by viscous damper," Structural Control and Health Monitoring, vol. 21, no. 2, pp. 205-224, 2014.

[54] K. Kim, J. Rye, and L. Chung, "Seismic performance of structures connected by viscoelastic dampers," Engineering Structures, vol. 28, no. 2, pp. 83-195, 2006.

[55] M. Basili and M. De Angelis, "Optimal passive control of adjacent structures interconnected with nonlinear hysteretic devices," Journal of Sound and Vibration, vol. 301, no. 1-2, pp. 106-125, 2007.

[56] O. Lavan, "A methodology for the integrated seismic design of nonlinear buildings with supplemental damping," Structural Control and Health Monitoring, vol. 22, no. 3, pp. 484-499, 2014.

[57] M. Martinez-Rodrigo and M. L. Romero, "An optimum retrofit strategy for moment resisting frames with nonlinear viscous dampers for seismic applications," Engineering Structures, vol. 25, no. 7, pp. 913-925, 2003.

[58] L. Keri and L. Curtis, "Analysis and design of inter-story isolation systems with nonlinear devices," Journal of Earthquake Engineering, vol. 14, no. 7, pp. 1044-1062, 2010. 
[59] D. Charmpis, P. Komodromos, and M. C. Phocas, "Optimized earthquake response of multi-storey buildings with seismic isolation at various levels," Earthquake Engineering \& Structural Dynamics, vol. 41, pp. 2289-2310, 2012.

[60] M. De Angelis, S. Perno, and A. Reggio, "Dynamic response and optimal design of structure with large mass ratio TMD," Earthquake Engineering \& Structural Dynamics, vol. 41, no. 1, pp. 41-60, 2012.

[61] G. W. Rodgers, J. G. Chase, J. B. Mander, N. C. Leach, and C. S. Denmead, "Experimental development, tradeoff analysis and design implementation of high force-to-volume damping technology," Bulletin of the New Zealand Society for Earthquake Engineering, vol. 40, no. 2, pp. 35-48, 2007.

[62] G. W. Rodgers, K. M. Solberg, J. G. Chase et al., "Performance of a damage-protected beam-column subassembly utilizing external HF2V energy dissipation devices," Earthquake Engineering \& Structural Dynamics, vol. 37, no. 13, pp. 15491564, 2008.

[63] T. Bacht, J. G. Chase, G. MacRae et al., "HF2V dissipator effects on the performance of a 3 story moment frame," Journal of Constructional Steel Research, vol. 67, no. 12, pp. 1843-1849, 2011.

[64] J. C. Golondrino, J. G. Chase, G. W. Rodgers, G. A. MacRae, and C. G. Clifton, "Velocity effects on the behaviour of highforce-to-volume lead dampers (HF2V) using different shaft configurations," in Proceedings of the 15th World Conference on Earthquake Engineering, Lisboa, Portugal, September 2012.

[65] J. E. Bobrow, F. Jabbari, and K. Thai, "An active truss element and control law for vibration suppression," Smart Materials and Structures, vol. 4, no. 4, pp. 264-269, 1995.

[66] F. Jabbari and J. E. Bobrow, "Vibration suppression with resettable device," Journal of Engineering Mechanics, vol. 128, no. 9, pp. 916-924, 2002.

[67] N. K. Hazaveh, J. G. Chase, G. W. Rodgers, and S. Pampanin, "Smart semi-active MR damper to control the structural response," Bulletin of the New Zealand Society for Earthquake Engineering, vol. 48, no. 4, pp. 235-245, 2015.

[68] J. G. Chase, K. J. Mulligan, A. Gue et al., "Re-shaping hysteretic behaviour using semi-active resettable device dampers," Engineering Structures, vol. 28, no. 10, pp. 14181429, 2006.

[69] G. W. Rodgers, J. B. Mander, J. Geoffrey Chase, K. J. Mulligan, B. L. Deam, and A. Carr, "Re-shaping hysteretic behaviour-spectral analysis and design equations for semi-active structures," Earthquake Engineering \& Structural Dynamics, vol. 36, no. 1, pp. 77-100, 2007.

[70] K. J. Mulligan, J. G. Chase, J. B. Mander et al., "Experimental validation of semi-active resetable actuators in a 1/5th scale test structure," Earthquake Engineering \& Structural Dynamics, vol. 38, no. 4, pp. 517-536, 2009.

[71] N. K. Hazaveh, G. W. Rodgers, J. G. Chase, and S. Pampanin, "Reshaping structural hysteresis response with semi-active viscous damping," Bulletin of Earthquake Engineering, vol. 15, no. 4, pp. 1789-1806, 2017.

[72] K. J. Mulligan, J. G. Chase, J. B. Mander, G. W. Rodgers, and R. B. Elliott, "Nonlinear models and validation for resetable device design and enhanced force capacity," Structural Control and Health Monitoring, vol. 17, no. 3, pp. 301-316, 2010.

[73] N. K. Hazaveh, G. W. Rodgers, J. G. Chase, and S. Pampanin, "Experimental test and validation of a direction- and displacement-dependent viscous damper," Journal of Engineering Mechanics, vol. 143, no. 11, p. 04017132, 2017.
[74] T. T. Soong and M. C. Costantinou, Passive and Active Structural Vibration Control in Civil Engineering, SpringerVerlag, Vienna, Austria, 2014.

[75] G. W. Housner, L. A. Bergman, T. K. Caughey et al., "Structural control: past, present, and future," Journal of Engineering Mechanics, vol. 123, no. 9, pp. 897-971, 1997.

[76] D. De Domenico, P. Fuschi, S. Pardo, and A. A. Pisano, "Strengthening of steel-reinforced concrete structural elements by externally bonded FRP sheets and evaluation of their load carrying capacity," Composite Structures, vol. 118, pp. 377-384, 2014.

[77] D. De Domenico, A. A. Pisano, and P. Fuschi, "Limit analysis on FRP-strengthened RC members," Frattura ed Integrità Strutturale, vol. 8, no. 29, pp. 209-221, 2014. 


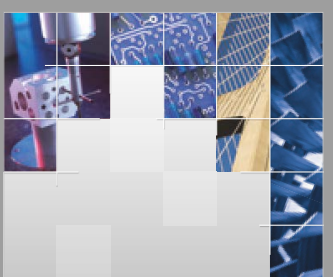

\section{Enfincering}
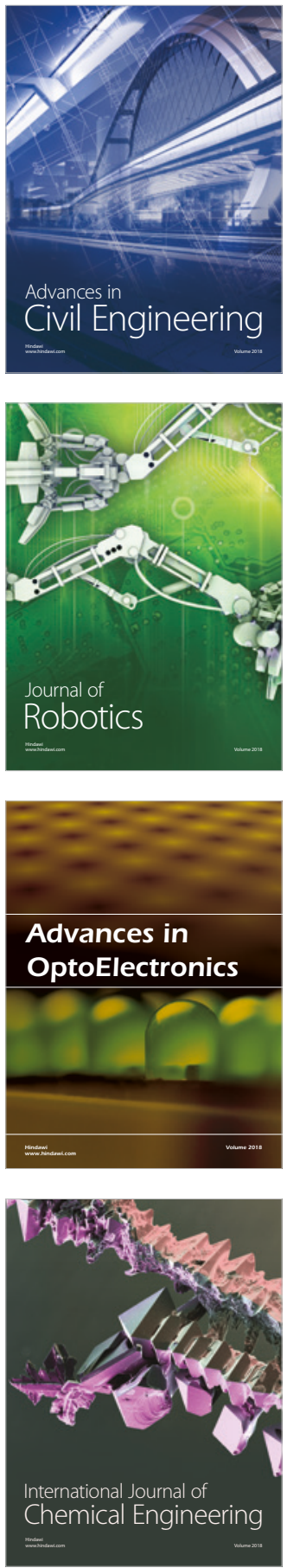

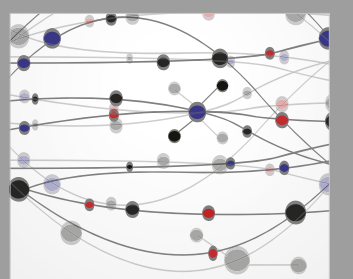

\section{Rotating \\ Machinery}

The Scientific World Journal

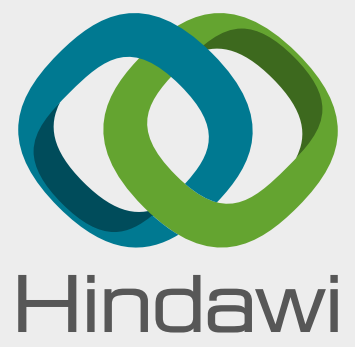

Submit your manuscripts at

www.hindawi.com
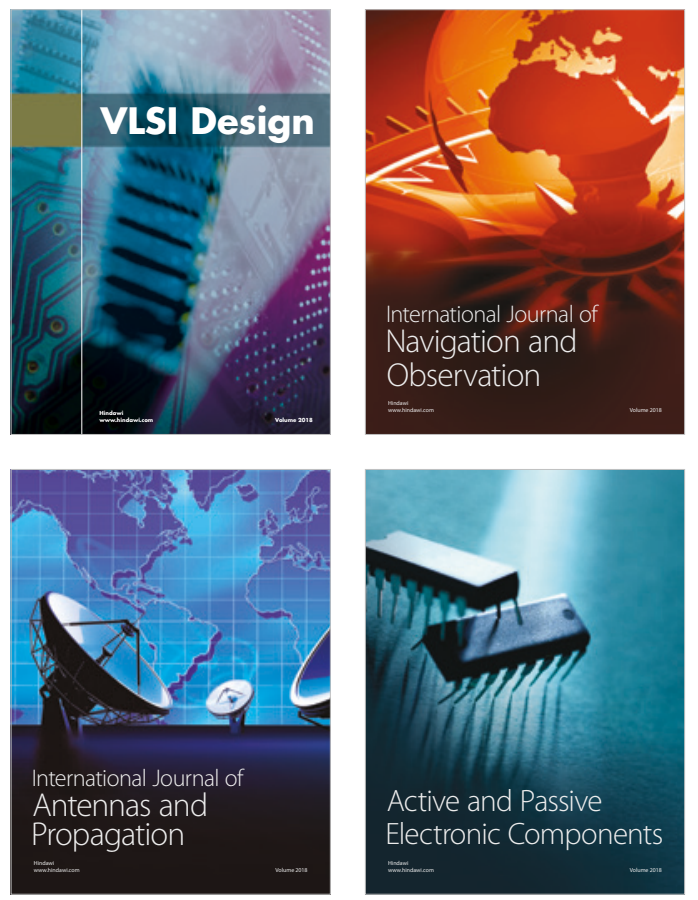
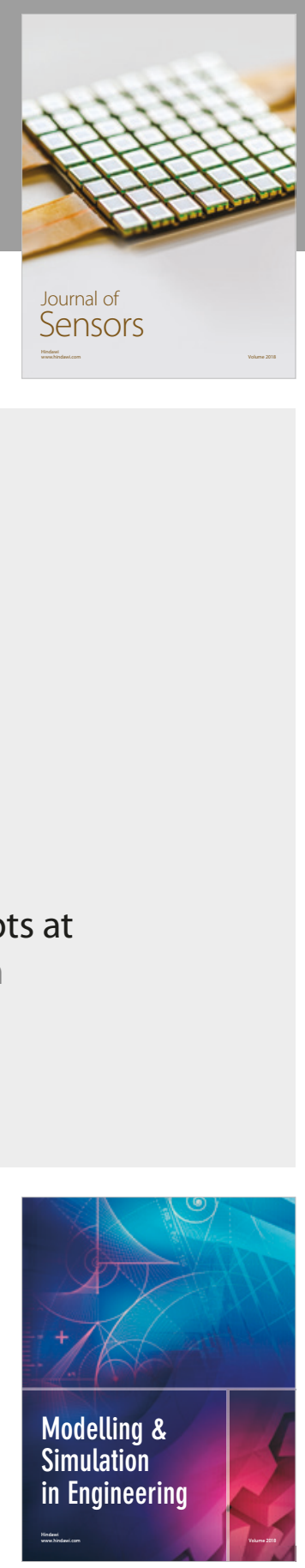

\section{Advances \\ Multimedia}
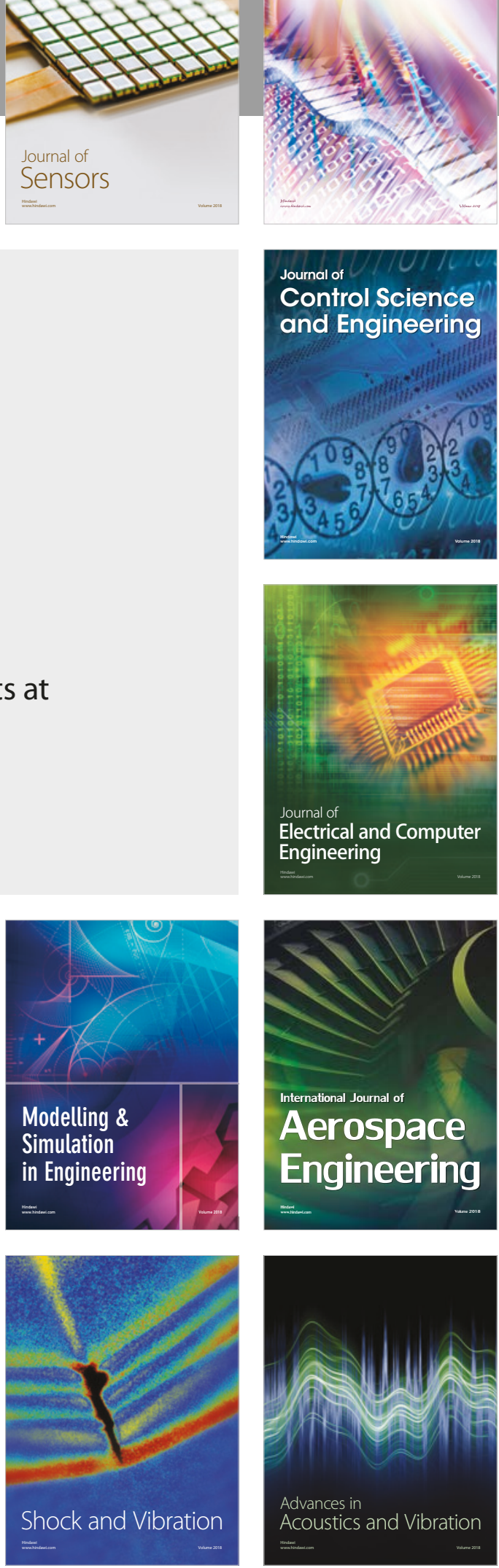